\title{
Adaptive Coding and Channel Shaping Through Reconfigurable Intelligent Surfaces: An Information-Theoretic Analysis
}

\author{
Roy Karasik ${ }^{\circledR}$, Graduate Student Member, IEEE, Osvaldo Simeone ${ }^{(}$, Fellow, IEEE, \\ Marco Di Renzo, Fellow, IEEE, and Shlomo Shamai (Shitz) ${ }^{\circledR}$, Life Fellow, IEEE
}

\begin{abstract}
A communication link aided by a reconfigurable intelligent surface (RIS) is studied in which the transmitter can control the state of the RIS via a finite-rate control link. Channel state information (CSI) is acquired at the receiver based on pilotassisted channel estimation, and it may or may not be shared with the transmitter. Considering quasi-static fading channels with imperfect CSI, capacity-achieving signalling is shown to implement joint encoding of the transmitted signal and of the response of the RIS. This demonstrates the information-theoretic optimality of RIS-based modulation, or "single-RF MIMO" systems. In addition, a novel signalling strategy based on separate layered encoding that enables practical successive cancellationtype decoding at the receiver is proposed. Numerical experiments show that the conventional scheme that fixes the reflection pattern of the RIS, irrespective of the transmitted information, as to maximize the achievable rate is strictly suboptimal, and is outperformed by the proposed adaptive coding strategies at all practical signal-to-noise ratio (SNR) levels.
\end{abstract}

Index Terms-RIS, imperfect CSI, single-RF MIMO, capacity.

\section{INTRODUCTION}

$\mathbf{I}$ $\mathrm{N}$ THE context of wireless communications, a reconfigurable intelligent surface (RIS) usually acts as an "anomalous mirror" or a "focusing lens" that can be configured to reflect or refract impinging radio waves towards arbitrary angles by applying appropriate phase shifts to the incident

Manuscript received December 1, 2020; revised April 23, 2021 and June 16 , 2021; accepted July 17, 2021. Date of publication July 27, 2021; date of current version November 18, 2021. This work was supported in part by the European Research Council (ERC) and in part by the Information and Communication Technologies (ICT) under the European Union's Horizon 2020 Research and Innovation Program under Grant 694630, Grant 725731, Grant 871464, and Grant 101017011. This article was presented in part at the 2020 IEEE International Symposium on Information Theory. The associate editor coordinating the review of this article and approving it for publication was J. Yuan. (Corresponding author: Roy Karasik.)

Roy Karasik and Shlomo Shamai (Shitz) are with the Department of Electrical and Computer Engineering, Technion-Israel Institute of Technology, Haifa 32000, Israel (e-mail: royk@ campus.technion.ac.il; sshlomo@ ee.technion.ac.il).

Osvaldo Simeone is with the Centre for Telecommunications Research, Department of Informatics, King's College London, London WC2R 2LS, U.K (e-mail: osvaldo.simeone@kcl.ac.uk).

Marco Di Renzo is with the Université Paris-Saclay, CNRS, CentraleSupélec, Laboratoire des Signaux et Systèmes, 91192 Gif-sur-Yvette, France (e-mail: marco.di-renzo@universite-paris-saclay.fr).

Color versions of one or more figures in this article are available at https://doi.org/10.1109/TCOMM.2021.3100621.

Digital Object Identifier 10.1109/TCOMM.2021.3100621

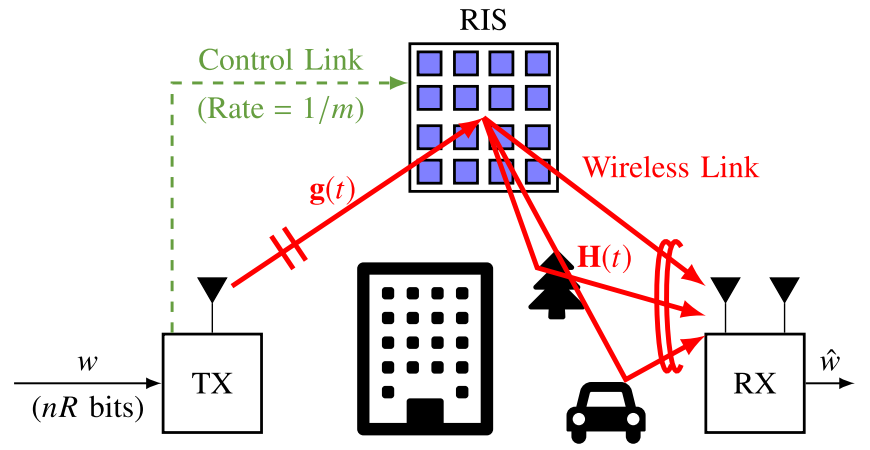

Fig. 1. Illustration of the network under study consisting of a single-RF transmitter (TX), a receiver (RX) with $N$ antennas, and an RIS with $K$ elements (in the figure, $N=2$ and $K=16$ ). The transmitter jointly encodes a message $w$ into a codeword of $n$ symbols, sent on the wireless link, and into a control action, sent on the control link to the RIS at a rate of one action every $m$ channel symbols. There is a strong line-of-sight between the transmitter and the RIS, whereas the reflected signal undergoes a multi-path channel.

signals [1], [2]. Due to these desirable properties, RISs are being considered for future wireless networks as means to shape the wireless propagation channel for signal, interference, security, and scattering engineering [3]-[7].

Most prior work, to be reviewed below, proposed to use the RIS as a fixed passive beamformer in order to control the SNR levels at the receivers. However, by altering the amplitude or phase of the incident signal, the RIS reflection pattern can also be jointly encoded with the transmitted signals as a function of the information message, thus enlarging the modulation space. One instantiation of this idea is the "single-RF MIMO" system introduced in [8] that encodes multiple information streams using the RIS reflection pattern and a single radio frequency (RF) chain [9].

While practical RIS-based modulation schemes exist [8]-[14], their information-theoretic properties have not been studied. This paper addresses this knowledge gap by studying the capacity of RIS-aided communication links in which a single-RF transmitter can control the state of an RIS via a finite-rate control link (see Fig. 1). The optimal configuration of the RIS requires knowledge of the CSI. The acquisition of CSI is made complicated by the fact that the RIS is a nearly-passive device, and hence it cannot process and transmit pilot signals. To account for this practical constraint, in this paper, the information-theoretic analysis is based on a model 
in which the CSI is estimated at the receiver via pilot-assisted transmission [15], and it may or may not be shared with the transmitter.

Related Work: The optimization of a fixed RIS reflection pattern has been studied in various scenarios. A comprehensive survey of the state-of-the-art is available in [1], and we mention here some representative examples. Algorithms for jointly optimizing precoding at the transmitter and beamforming at the RIS were proposed for a point-to-point Multiple-Input Single-Output (MISO) systems in [16], and for Multiple-Input Multiple-Output (MIMO) systems in [17], [18]. RIS-based passive beamforming was compared to conventional relaying methods such as amplify-and-forward and decode-and-forward in [2].

Acquiring CSI is crucial for RIS-aided communication. Channel estimation schemes were proposed in [15], [19], in which RIS training patterns are designed under the constraint of discrete phase shifts. The overhead required for channel estimation was studied in [20], and an overheadaware resource allocation framework was developed. Channel estimation based on statistical CSI is used in [21] to reduce the channel training overhead.

Schemes for encoding information in the configuration of the RIS have been recently presented. In [10]-[13], information is encoded in the reflection patterns of the RIS by setting the amplitude of each reflecting element to be 0 or 1 . In [14], the receive antenna for which the SNR is maximized encodes the information bits using index modulation [22]. The strategies above are extended in [8] by implementing phase-shift keying (PSK) and quadrature amplitude modulation (QAM) at each element, and by using two independent data streams to control the RIS.

Main Contributions: This work provides an informationtheoretic analysis of the RIS-aided system illustrated in Fig. 1, which consists of a single-RF transmitter and a receiver with $N$ antennas. CSI is assumed to be acquired at the receiver via pilot-based transmission, and it may or may not be shared with the transmitter. We first derive the capacity for any RIS control rate, and prove that jointly encoding data onto the transmitted signals and RIS reflection pattern is generally necessary to achieve the maximum information rate. We explicitly characterize the performance gain of joint encoding in the high-SNR regime. Then, we propose an achievable scheme based on layered encoding and successive cancellation decoding (SCD) that enables RIS-based modulation, while supporting standard separate encoding and decoding strategies. Numerical experiments demonstrate that, for SNR levels of practical interest and for a sufficiently fast RIS control link, capacity-achieving joint encoding provides significant gain over the max-SNR approach, which fixes the reflection pattern. However, joint encoding is shown to require a more accurate channel estimation compared to the max-SNR scheme, and is hence mostly desirable for long channel coherence blocks. The results in this paper were partially presented in [23], which only considers perfect CSI at the transmitter and receiver.

Organization: The rest of the paper is organized as follows. In Section II, we present an information-theoretic model for an RIS-aided quasi-static fading channel with imperfect CSI obtained via channel estimation. In Section III, we derive the capacity and we compare it to the rates achieved by two standard suboptimal signalling schemes: a max-SNR scheme that does not encode information in the RIS reflection pattern, and an RIS-based signalling scheme that modulates the reflection pattern uniformly and has no beamforming gain. In Section IV, we describe an achievable strategy based on layered encoding and successive cancellation decoding with basic separate encoding and decoding procedures. In Section V, lower bounds on the capacity and achievable rates are derived. In Section VI, we present numerical results in order to compare the capacity with the rates achieved by the suboptimal strategies, and to assess the impact of imperfect CSI on performance. Finally, in Section VII, we conclude the paper and highlight some open problems.

Notation: Random variables, vectors, and matrices are denoted by lowercase, boldface lowercase, and boldface uppercase Roman-font letters, respectively. Realizations of random variables, vectors, and matrices are denoted by lowercase, boldface lowercase, and boldface uppercase italic-font letters, respectively. For example, $x$ is a realization of random variable $\mathrm{x}, \boldsymbol{x}$ is a realization of random vector $\mathbf{x}$, and $\boldsymbol{X}$ is a realization of random matrix $\mathbf{X}$. For any positive integer $K$, we define the set $[K] \triangleq\{1,2, \ldots, K\}$. The cardinality of a set $\mathcal{A}$ is denoted as $|\mathcal{A}|$. The Mahalanobis norm of vector $\boldsymbol{v}$ with positive semidefinite matrix $\boldsymbol{S}$ is defined as $\|\boldsymbol{v}\|_{S} \triangleq \sqrt{\boldsymbol{v}^{*} \boldsymbol{S}^{-1} \boldsymbol{v}}$, where $\boldsymbol{v}^{*}$ denotes the conjugate transpose of vector $\boldsymbol{v}$, and the $\ell^{2}$-norm of a vector $\boldsymbol{v}$ is denoted as $\|\boldsymbol{v}\|$. $\operatorname{diag}(\boldsymbol{x})$ represents a diagonal matrix with diagonal given by the vector $\boldsymbol{x}$. The trace of a matrix $\boldsymbol{X}$ is denoted as $\operatorname{tr}(\boldsymbol{X})$. The vectorization of matrix $\boldsymbol{H}$, i.e., the operator that stacks the columns of $\boldsymbol{H}$ on top of one another, is denoted by $\operatorname{vec}(\boldsymbol{H})$. The Kronecker product of matrices $\boldsymbol{A}$ and $\boldsymbol{B}$ is denoted by $\boldsymbol{A} \otimes \boldsymbol{B}$.

\section{SYSTEM MODEL}

We consider the system depicted in Fig. 1 in which a singleRF transmitter communicates with a receiver equipped with $N$ antennas over a quasi-static fading channel in the presence of an RIS that comprises $K$ nearly-passive reconfigurable elements. The $K$ reconfigurable elements are spaced half of the wavelength apart, so that the mutual coupling or channel correlation effects can be ignored as a first-order approximation [24]. We explore the potential improvement in capacity that can be obtained when the transmitter can encode its message $w \in\left[2^{n R}\right]$ of rate $R$ [bits/symbol] not only into a codeword of $n$ symbols sent on the wireless link to the receiver, but also in the reflection pattern of the RIS. The reflection pattern is controlled through a rate-limited control link, and is defined by the phase shifts that each of the $K$ RIS elements applies to the impinging wireless signal. Note that joint encoding takes place at the transmitter, i.e., no information processing capabilities are required for the RIS, which is assumed to be a nearly-passive device.

As illustrated in Fig. 2, the fading coefficients are assumed to remain constant for a coherence interval of $T$ symbol periods, after which they change to new independent values. The coding slot of $n$ symbols hence contains $n / T$ coherence blocks, which is taken to be an integer. The codeword transmitted in a coding slot has $n$ symbols from a constellation $\mathcal{S}$ of $S=|\mathcal{S}|$ points. The constellation $\mathcal{S}$ is assumed to have an 
coding slot ( $n$ symbols)

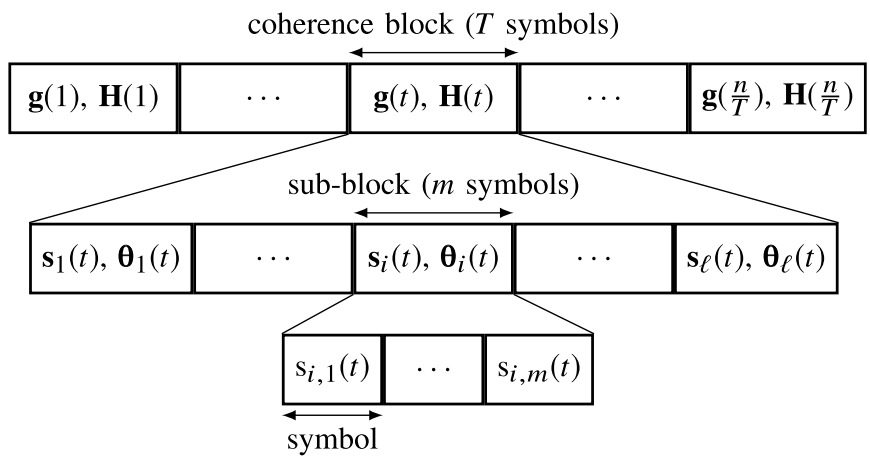

Fig. 2. Illustration of a coding slot. Each slot consists of $n / T$ coherence blocks, which, due to the RIS control link rate, contain $\ell$ sub-blocks of $m$ symbols each.

average power of one, i.e.

$$
\frac{1}{S} \sum_{s \in \mathcal{S}}|s|^{2}=1 .
$$

The phase shift applied by each element of the RIS is chosen from a finite set $\mathcal{A}$ of $A=|\mathcal{A}|$ distinct hardware-determined values. The RIS is controlled by the transmitter by selecting the $K$ phases of the elements as a function of the message $w$. Due to practical limitations on the RIS configuration rate, we assume that the phase shifts can only be modified once for each sub-block that comprises $m$ consecutive transmitted symbols. As illustrated in Fig. 2, we assume that each coherence block contains $\ell=T / m$ sub-blocks for some integer $\ell \geq 1$, i.e., the RIS can be configured at the beginning of each subblock $i \in[\ell]$ of $m$ transmitted symbols. Note that if $\ell=1$, i.e., if $m=T$, the reflection pattern of the RIS is fixed for the entire coherence block.

The channel from the transmitter to the RIS in the $t$ th coherence block, $t \in[n / T]$, is denoted by the vector $\mathbf{g}(t) \in$ $\mathbb{C}^{K \times 1}$, and the channel from the RIS to the $N$ receiving antennas is denoted by the matrix $\mathbf{H}(t) \in \mathbb{C}^{N \times K}$. In order to support multiple information streams with a single $\mathrm{RF}$ chain, the transmitter and RIS are expected to be placed such that there is a strong line-of-sight between them [9], [14]. Therefore, we assume that the elements of the channel vector $\mathrm{g}(t)$ have random phases and unit amplitude, as illustrated in Fig. 1. In contrast, the reflected signal is assumed to undergo a multi-path channel before being received, and hence the elements of the matrix $\mathbf{H}(t)$ are independent and identically distributed (i.i.d.) as $\mathcal{C N}(0,1)$. Moreover, as in, e.g., [8], [14], we assume that the direct link between transmitter and receiver is blocked, so that the propagation from transmitter to receiver occurs solely through the reflected signal from the RIS. During the $t$ th coherence block, the fraction of the codeword consisting of $m$ symbols transmitted in the $i$ th subblock, $i \in[\ell]$, is denoted by $\mathbf{s}_{i}(t)=\left(\mathrm{s}_{i, 1}(t), \ldots, \mathrm{s}_{i, m}(t)\right)^{\top} \in$ $\mathcal{S}^{m \times 1}$, and is assumed to satisfy

$$
\frac{1}{m} \mathbb{E}\left[\mathbf{s}_{i}^{*}(t) \mathbf{s}_{i}(t)\right] \leq 1 \text {. }
$$

The phase shifts applied by the RIS in the $i$ th sub-block are denoted by the vector

$$
e^{j \boldsymbol{\theta}_{i}(t)} \triangleq\left(e^{j \theta_{i, 1}(t)}, \ldots, e^{j \theta_{i, K}(t)}\right)^{\top}
$$

with $\theta_{i, k}(t) \in \mathcal{A}$ being the phase shift applied by the $k$ th RIS element, $k \in[K]$. Finally, we denote the signal received by the $N$ antennas for the $q$ th transmitted symbol by $\mathbf{y}_{i, q}(t) \in$ $\mathbb{C}^{N \times 1}, q \in[m]$. The overall received signal matrix $\mathbf{Y}_{i}(t)=$ $\left(\mathbf{y}_{i, 1}(t), \ldots, \mathbf{y}_{i, m}(t)\right) \in \mathbb{C}^{N \times m}$ in the $i$ th sub-block can hence be written as

$$
\begin{aligned}
\mathbf{Y}_{i}(t) & =\mathbf{H}(t) \operatorname{diag}\left(e^{j \boldsymbol{\theta}_{i}(t)}\right) \mathbf{g}(t) \gamma_{i}(t) \mathbf{s}_{i}^{\boldsymbol{\top}}(t)+\mathbf{Z}_{i}(t) \\
& =\overline{\mathbf{H}}(t) e^{j \boldsymbol{\theta}_{i}(t)} \gamma_{i}(t) \mathbf{s}_{i}^{\boldsymbol{\top}}(t)+\mathbf{Z}_{i}(t),
\end{aligned}
$$

where the matrix $\overline{\mathbf{H}}(t) \triangleq \mathbf{H}(t) \operatorname{diag}(\mathbf{g}(t))$, whose elements are i.i.d. $\mathcal{C N}(0,1)$, combines the channels $\mathbf{g}(t)$ and $\mathbf{H}(t)$; the scalar $\gamma_{i}(t)>0$ denotes the power gain applied to the transmitted signal $\mathbf{s}_{i}(t)$, which is subject to the power constraint

$$
\frac{1}{\ell} \sum_{i=1}^{\ell} \gamma_{i}^{2}(t)=P
$$

for some $P>0$; and the matrix $\mathbf{Z}_{i}(t) \in \mathbb{C}^{N \times m}$, whose elements are i.i.d. as $\mathcal{C} \mathcal{N}(0,1)$, denotes the additive white Gaussian noise at the receiving antennas. It is worth noting that the product $\overline{\mathbf{H}}(t) e^{j \boldsymbol{\theta}_{i}(t)}$ in (4) can be viewed as an augmented channel, shaped by the RIS for increasing the capacity.

Since the message $w$ is encoded onto both transmitted symbols $\mathbf{s}_{i}(t)$ and phase shifts $\boldsymbol{\theta}_{i}(t), i \in[\ell], t \in[n / T]$, we denote the effective channel input as

$$
\overline{\mathbf{X}}_{i}(t) \triangleq e^{j \boldsymbol{\theta}_{i}(t)} \mathbf{s}_{i}^{\top}(t) .
$$

With this notation, the channel (4) can be restated as

$$
\mathbf{Y}_{i}(t)=\gamma_{i}(t) \overline{\mathbf{H}}(t) \overline{\mathbf{X}}_{i}(t)+\mathbf{Z}_{i}(t) .
$$

At first glance, the channel (7) resembles a standard multiple-antenna wireless communication link [25]. In (7), however, the input matrix $\overline{\mathbf{X}}_{i}(t)$ is rank-one and is chosen from the finite set

$$
\begin{aligned}
\mathcal{C} \triangleq\left\{\overline{\boldsymbol{X}}: \overline{\boldsymbol{X}}=\left(e^{j \theta_{1}}, \ldots, e^{j \theta_{K}}\right)^{\boldsymbol{\top}} \boldsymbol{s}^{\boldsymbol{\top}},\right. & \\
\boldsymbol{s} & \left.\in \mathcal{S}^{m \times 1}, \boldsymbol{\theta} \in \mathcal{A}^{K \times 1}\right\} .
\end{aligned}
$$

As a special case, for a fixed RIS reflection pattern $\boldsymbol{\theta}_{i}=\boldsymbol{\theta}$ for all $i \in[\ell]$, i.e., when the same phase shift vector is used for the entire coherence block, the channel input is chosen from the subset

$$
\mathcal{C}(\boldsymbol{\theta}) \triangleq\left\{\overline{\boldsymbol{X}}: \overline{\boldsymbol{X}}=\left(e^{j \theta_{1}}, \ldots, e^{j \theta_{K}}\right)^{\top} \boldsymbol{s}^{\boldsymbol{\top}}, \boldsymbol{s} \in \mathcal{S}^{m \times 1}\right\} .
$$

In the present paper, we study the impact of imperfect CSI on the achievable rates. In order to characterize the joint distribution of channel estimation and output signal, we vectorize the channel matrix $\overline{\mathbf{H}}(t)$ and output $\mathbf{Y}_{i}(t)$ in (7) as

$$
\overline{\mathbf{h}}(t) \triangleq \operatorname{vec}(\overline{\mathbf{H}}(t))
$$

and

$$
\mathbf{y}_{i}(t) \triangleq \operatorname{vec}\left(\mathbf{Y}_{i}(t)\right)=\gamma_{i}(t) \overline{\mathbf{X}}_{i}^{\otimes}(t) \overline{\mathbf{h}}(t)+\mathbf{z}_{i}(t),
$$

respectively, where we have defined the vector $\mathbf{z}_{i}(t) \triangleq$ $\operatorname{vec}\left(\mathbf{Z}_{i}(t)\right) \in \mathbb{C}^{N m \times 1}$, and, for any matrix $\overline{\mathbf{X}}$, the matrix $\overline{\mathbf{X}}^{\otimes}$ is defined as the Kronecker product

$$
\overline{\mathbf{X}}^{\otimes} \triangleq \overline{\mathbf{X}}^{\top} \otimes \boldsymbol{I}_{N}
$$




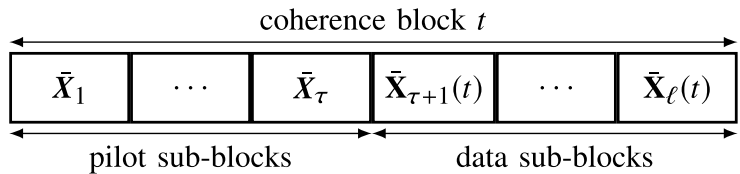

Fig. 3. Structure of a coherence block. The first $\tau$ sub-blocks in each coherence block are used for channel estimation.

\section{A. Training and Channel Estimation}

As illustrated in Fig. 3, we focus our attention on transmission schemes in which, for each coherence block $t \in[n / T]$, the first $\tau \geq 0$ sub-blocks are used to transmit pilot symbols known to the receiver. That is, we have

$$
\overline{\mathbf{X}}_{i}(t)=\overline{\boldsymbol{X}}_{i}, \quad \forall i \in[\tau], t \in[n / T],
$$

where $\overline{\boldsymbol{X}}_{1}, \ldots, \overline{\boldsymbol{X}}_{\tau}$ denote the pilot symbols. The pilot symbols satisfy the power constraint

$$
\operatorname{tr}\left(\boldsymbol{X}_{1: \tau} \boldsymbol{X}_{1: \tau}^{*}\right) \leq K m \tau,
$$

where we have defined matrix

$$
\boldsymbol{X}_{1: \tau} \triangleq\left(\overline{\boldsymbol{X}}_{1}, \ldots, \overline{\boldsymbol{X}}_{\tau}\right) \in \mathcal{C}^{1 \times \tau} .
$$

As for the transmitter, we assume that either it has no access to the CSI or that it has access to the receiver's CSI via a feedback channel.

The transmission power can vary between the training and information transmission phases. Accordingly, the power gain $\gamma_{i}(t)$ in (4) has two levels

$$
\gamma_{i}(t)=\left\{\begin{array}{l}
\gamma_{\tau} \text { for } 1 \leq i \leq \tau \\
\gamma_{d} \text { for } \tau+1 \leq i \leq \ell
\end{array}\right.
$$

The power constraint (5) can hence be restated as

$$
\frac{\tau}{\ell} \gamma_{\tau}^{2}+\frac{\ell-\tau}{\ell} \gamma_{d}^{2}=P
$$

Therefore, the vectorized channel output during the training phase is

$\mathbf{y}_{1: \tau}(t) \triangleq\left(\mathbf{y}_{1}^{\top}(t), \ldots, \mathbf{y}_{\tau}^{\top}(t)\right)^{\top}=\gamma_{\tau} \boldsymbol{X}_{1: \tau}^{\otimes} \overline{\mathbf{h}}(t)+\mathbf{z}_{1: \tau}(t)$,

with $\mathbf{z}_{1: \tau}(t) \triangleq\left(\mathbf{z}_{1}^{\top}(t), \ldots, \mathbf{z}_{\tau}^{\top}(t)\right)^{\boldsymbol{\top}} \in \mathbb{C}^{N m \tau \times 1}$.

Based on the pilot symbols $\boldsymbol{X}_{1: \tau}$, the receiver estimates the channel vector $\mathbf{h}(t)$ using the minimum mean-square error (MMSE) estimator, which yields $\hat{\mathbf{h}}(t)=\mathbb{E}\left[\overline{\mathbf{h}}(t) \mid \mathbf{y}_{1: \tau}(t)\right]$ as the estimate of $\overline{\mathbf{h}}(t)$ from the observations $\mathbf{y}_{1: \tau}(t)$. Since vectors $\overline{\mathbf{h}}(t)$ and $\mathbf{y}_{1: \tau}(t)$ are jointly Gaussian distributed, the MMSE estimator can be computed as the linear MMSE estimator [26], i.e.,

$$
\hat{\mathbf{h}}(t)=\gamma_{\tau}\left(\boldsymbol{X}_{1: \tau}^{\otimes}\right)^{*}\left(\gamma_{\tau}^{2} \boldsymbol{X}_{1: \tau}^{\otimes}\left(\boldsymbol{X}_{1: \tau}^{\otimes}\right)^{*}+\boldsymbol{I}_{N m \tau}\right)^{-1} \mathbf{y}_{1: \tau}(t),
$$

and the estimation error is a Gaussian random vector whose covariance matrix is

$$
\begin{aligned}
\boldsymbol{\Gamma}_{\mathrm{MMSE}} \triangleq & \mathbb{E}\left[(\overline{\mathbf{h}}(t)-\hat{\mathbf{h}}(t))(\overline{\mathbf{h}}(t)-\hat{\mathbf{h}}(t))^{*}\right] \\
= & \boldsymbol{I}_{N K} \\
& -\gamma_{\tau}^{2}\left(\boldsymbol{X}_{1: \tau}^{\otimes}\right)^{*}\left(\gamma_{\tau}^{2} \boldsymbol{X}_{1: \tau}^{\otimes}\left(\boldsymbol{X}_{1: \tau}^{\otimes}\right)^{*}+\boldsymbol{I}_{N m \tau}\right)^{-1} \boldsymbol{X}_{1: \tau}^{\otimes} .
\end{aligned}
$$

In order to assess how channel estimation affects the achievable performance, we shall also consider as a benchmark the case of perfect CSI, which corresponds to the case study in which the vector $\hat{\mathbf{h}}(t)=\overline{\mathbf{h}}(t)$ is available to both the transmitter and receiver as side information without any training $(\tau=0)$.

\section{B. Channel Encoding}

As discussed, in each coherence block, the transmitter selects the $\ell-\tau$ data sub-blocks

$$
\mathbf{X}(t) \triangleq\left(\overline{\mathbf{X}}_{\tau+1}(t), \ldots, \overline{\mathbf{X}}_{\ell}(t)\right) \in \mathcal{C}^{1 \times(\ell-\tau)}
$$

based on the information message $w$ and the channel estimate $\hat{\mathbf{h}}(t)$, if available. The vectorized channel output in (11), received over the $\ell-\tau$ data sub-blocks, can be expressed as

$$
\mathbf{y}(t) \triangleq\left(\mathbf{y}_{\tau+1}^{\top}(t), \ldots, \mathbf{y}_{\ell}^{\top}(t)\right)^{\boldsymbol{\top}}=\gamma_{d} \mathbf{X}^{\otimes}(t) \overline{\mathbf{h}}(t)+\mathbf{z}(t),
$$

with $\mathbf{z}(t) \triangleq\left(\mathbf{z}_{\tau+1}^{\top}(t), \ldots, \mathbf{z}_{\ell}^{\top}(t)\right)^{\top} \in \mathbb{C}^{N m(\ell-\tau) \times 1}$. Having received the vector $\mathbf{y}(t)$ in (22) for $t \in[n / T]$, the decoder produces the estimate $\hat{w}=\hat{w}(\mathbf{y}(1), \ldots, \mathbf{y}(n / T), \mathcal{H})$ based on the channel estimates $\mathcal{H} \triangleq\{\hat{\mathbf{h}}(1), \ldots, \hat{\mathbf{h}}(n / T)\}$ in (19).

For a specific choice of training parameters $\tau, \gamma_{\tau}$, and $\boldsymbol{X}_{1: \tau}$, a rate $R\left(\tau, \gamma_{\tau}, \boldsymbol{X}_{1: \tau}\right)$ is said to be achievable if the probability of error satisfies the limit $\operatorname{Pr}(\hat{w} \neq w) \rightarrow 0$ when the codeword length grows large, i.e., $n \rightarrow \infty$. The corresponding ergodic capacity $C\left(\tau, \gamma_{\tau}, \boldsymbol{X}_{1: \tau}\right)$ is defined as the maximum over all achievable rates, i.e.,

$$
\begin{aligned}
& C\left(\tau, \gamma_{\tau}, \boldsymbol{X}_{1: \tau}\right) \\
& \triangleq \sup \left\{R\left(\tau, \gamma_{\tau}, \boldsymbol{X}_{1: \tau}\right): R\left(\tau, \gamma_{\tau}, \boldsymbol{X}_{1: \tau}\right) \text { is achievable }\right\},
\end{aligned}
$$

where the supremum is taken over all joint encoding and decoding schemes. The number of sub-blocks used for training $0 \leq \tau \leq \ell$, pilot symbols $\boldsymbol{X}_{1: \tau}$, and power-amplifier gain $\gamma_{\tau}>0$ can all be optimized to increase the achievable rate.

\section{Channel Capacity}

In this section, we derive the capacity $C\left(\tau, \gamma_{\tau}, \boldsymbol{X}_{1: \tau}\right)$ defined in (23) and we prove that the conventional scheme that does not encode information in the RIS reflection pattern is strictly suboptimal. More specifically, this result is proved in the high-SNR regime by characterizing the gain of the proposed joint encoding. For finite values of the SNR, on the other hand, the performance gain is evaluated in Section VI via numerical experiments.

Most works on RIS-aided systems consider Gaussian codebooks for the transmitted signal $\mathbf{s}_{i}(t)$. This implies that the resulting achievable rates are formulated in the standard form $\log _{2}(1+$ SNR $)$, even in the presence of imperfect CSI by using standard bounds [27]. In contrast, as described in Section II, we focus our attention on the more practical model in which the transmitted symbols and the RIS elements' phase response take values from finite sets. As a result, standard capacity expressions of the form $\log _{2}(1+\mathrm{SNR})$ are not applicable, and standard techniques for bounding the capacity under imperfect CSI cannot be used. Specifically, lower bounding the capacity by modeling the residual channel estimation noise as Gaussian [28], [29] does not hold for finite input constellations [30]. Therefore, the expressions for the capacity and achievable rates that we present in this section are more complex, and require the following definitions. 
Definition 1: The cumulant-generating function (CGF) of a random variable $\mathrm{u}$ is defined as

$$
\kappa_{r}(\mathrm{u}) \triangleq \log _{2}\left(\mathbb{E}\left[e^{r \mathrm{u}}\right]\right), \quad r \in \mathbb{R} .
$$

The value of the CGF for $r=1$ is denoted by $\kappa(\mathrm{u}) \triangleq \kappa_{1}(\mathrm{u})$.

Definition 2: The CGF of a random variable u conditioned on a random vector $\mathbf{x}$ is defined as

$$
\kappa_{r}(\mathrm{u} \mid \mathbf{x}) \triangleq \mathbb{E}\left[\log _{2}\left(\mathbb{E}\left[e^{r \mathrm{u}} \mid \mathbf{x}\right]\right)\right], \quad r \in \mathbb{R} .
$$

The value of the conditional CGF for $r=1$ is denoted by $\kappa(\mathrm{u} \mid \mathbf{x}) \triangleq \kappa_{1}(\mathrm{u} \mid \mathbf{x})$.

We now derive the capacity for the general case with imperfect CSI available at both the transmitter and receiver. In particular, the capacity is formulated in the form of an optimization problem with respect to the encoding distribution $p_{\mathbf{X} \mid \hat{\mathbf{h}}}(\boldsymbol{X} \mid \hat{\boldsymbol{h}})$ of the effective inputs in (21) given the channel estimate $\hat{\mathbf{h}}$. To this end, we define the covariance matrix of the received signal $\mathbf{y}(t)$ in (22) conditioned on the channel estimate $\hat{\mathbf{h}}(t)$ and the input $\mathbf{X}(t)$ as

$$
\begin{aligned}
\mathbb{E}\left[\mathbf{y}(t) \mathbf{y}(t)^{*} \mid \hat{\mathbf{h}}(t), \mathbf{X}(t)\right]= & \boldsymbol{I}_{N m(\ell-\tau)} \\
& +\gamma_{d}^{2} \mathbf{X}^{\otimes}(t) \cdot \boldsymbol{\Gamma}_{\mathrm{MMSE}} \cdot\left(\mathbf{X}^{\otimes}(t)\right)^{*} \\
= & \boldsymbol{\Gamma}(\mathbf{X}(t)),
\end{aligned}
$$

where, for any matrix $\mathbf{X}$, we have defined the positive semidefinite matrix $\boldsymbol{\Gamma}(\mathbf{X})$ as

$$
\boldsymbol{\Gamma}(\mathbf{X}) \triangleq \boldsymbol{I}_{N m(\ell-\tau)}+\gamma_{d}^{2} \mathbf{X}^{\otimes} \cdot \boldsymbol{\Gamma}_{\mathrm{MMSE}} \cdot\left(\mathbf{X}^{\otimes}\right)^{*},
$$

with matrix $\Gamma_{\text {MMSE }}$ defined in (20). We also define the decomposition

$$
\Gamma(\mathbf{X})=\boldsymbol{V}(\mathbf{X}) \boldsymbol{V}(\mathbf{X})^{*},
$$

where $\boldsymbol{V}(\mathbf{X})$ is a square root matrix of $\boldsymbol{\Gamma}(\mathbf{X})$.

Proposition 1: When the MMSE estimate $\hat{\mathbf{h}}(t)$ in (19) is available at both the receiver and transmitter, the capacity of the channel (22) is given as

$$
\begin{aligned}
C\left(\tau, \gamma_{\tau}, \boldsymbol{X}_{1: \tau}\right)= & -\frac{N(\ell-\tau)}{\ell} \log _{2}(e) \\
& -\min _{\substack{p_{\mathbf{X}}(\mathbf{\hat { h }} \mid \hat{\boldsymbol{h}}): \\
\mathbb{E}\left[\operatorname{tr}\left(\mathbf{X} \mathbf{X}^{*}\right)\right] \leq K m(\ell-\tau), \mathbf{X} \in \mathcal{C}^{1 \times(\ell-\tau)}}} \frac{1}{m \ell} \kappa\left(\mathrm{u} \mid \mathbf{X}_{1}, \mathbf{z}, \hat{\mathbf{h}}\right), \\
&
\end{aligned}
$$

where the random variable $\mathrm{u}$ is defined as

$\mathrm{u} \triangleq \ln \left(\frac{\left|\boldsymbol{\Gamma}\left(\mathbf{X}_{1}\right)\right|}{\left|\boldsymbol{\Gamma}\left(\mathbf{X}_{2}\right)\right|}\right)-\left\|\boldsymbol{V}\left(\mathbf{X}_{1}\right) \mathbf{z}+\gamma_{d}\left(\mathbf{X}_{1}^{\otimes}-\mathbf{X}_{2}^{\otimes}\right) \hat{\mathbf{h}}\right\|_{\boldsymbol{\Gamma}\left(\mathbf{X}_{2}\right)}^{2}$

with independent random vectors $\mathbf{z} \sim \mathcal{C N}\left(\mathbf{0}, \boldsymbol{I}_{N m(\ell-\tau)}\right)$ and $\hat{\mathbf{h}} \sim \mathcal{C N}\left(\mathbf{0}, \boldsymbol{I}_{N K}-\boldsymbol{\Gamma}_{\mathrm{MMSE}}\right)$, and random matrices $\mathbf{X}_{1}, \mathbf{X}_{2} \sim$ $p_{\mathbf{X} \mid \hat{\mathbf{h}}}(\boldsymbol{X} \mid \hat{\boldsymbol{h}})$ that are conditionally independent given $\hat{\mathbf{h}}$. Furthermore, for $\tau \geq K$, we have the high-SNR limit

$$
\lim _{P \rightarrow \infty} C\left(\tau, \gamma_{\tau}, \boldsymbol{X}_{1: \tau}\right)=\frac{(\ell-\tau) \log _{2}(|\mathcal{C}|)}{m \ell},
$$

which, for a given cardinality $S=|\mathcal{S}|$ of the signal constellation, is maximized if the amplitude shift keying (ASK) modulation is used, i.e.,

$$
\mathcal{S}=\{\sigma, 3 \sigma, \ldots,(2 S-1) \sigma\},
$$

where the factor $\sigma \triangleq \sqrt{3 /\left[3+4\left(S^{2}-1\right)\right]}$ ensures a unit average power constraint. In this case, the high-SNR limit is

$\lim _{P \rightarrow \infty} C\left(\tau, \gamma_{\tau}, \boldsymbol{X}_{1: \tau}\right)=\frac{\ell-\tau}{m \ell}\left[m \log _{2}(S)+K \log _{2}(A)\right]$.

Proof: See Appendix A.

Achieving the capacity in (29) generally requires joint encoding over the codeword symbols $\mathbf{s}_{i}(t)$ and RIS reflection variables $\boldsymbol{\theta}_{i}(t)$, for all data sub-blocks $i=\tau+1, \ldots, \ell$, $t \in[n / T]$, as well as joint decoding of the message $w$ at the receiver based on the information encoded over both $\mathbf{s}_{i}(t)$ and $\boldsymbol{\theta}_{i}(t)$. In (29), this is specified in the optimization over the distribution $p_{\mathbf{X} \mid \hat{\mathbf{h}}}(\boldsymbol{X} \mid \hat{\boldsymbol{h}})$ of the input $\mathbf{X}(t)=$ $\left(\overline{\mathbf{X}}_{\tau+1}(t), \ldots, \overline{\mathbf{X}}_{\ell}(t)\right)$ in (21), which, by (6), is a function of both $\mathbf{s}_{i}(t)$ and $\boldsymbol{\theta}_{i}(t)$. However, the high-SNR asymptotic limit in (31) implies that, in the high-SNR regime, capacity is achieved by using independent random codebooks with uniform distribution for the codeword symbols $\mathbf{s}$ and the RIS reflection pattern $\theta$, and perfect channel estimation can be obtained by using $\tau \geq K$ pilot sub-blocks.

At a computational level, problem (29) is convex (see Appendix A), and hence it can be solved by using convex optimization tools. Moreover, calculating $\kappa\left(\mathrm{u} \mid \mathbf{X}_{1}, \mathbf{z}, \hat{\mathbf{h}}\right)$ in (29) involves evaluating the expectation over the random vectors $\mathbf{z}$ and $\hat{\mathbf{h}}$, and over the random matrices $\mathbf{X}_{1}$ and $\mathbf{X}_{2}$. Since $\mathbf{z}$ and $\hat{\mathbf{h}}$ are continuous random vectors, the former expectation may be estimated via an empirical average, while the second requires summing over $|\mathcal{C}|^{\ell-\tau}$ terms.

The following two corollaries formulate the capacity under the assumption of imperfect CSI available only at the receiver, and under the assumption of perfect CSI available at both the transmitter and receiver, respectively.

Corollary 1: When the MMSE estimate $\hat{\mathbf{h}}(t)$ in (19) is available only at the receiver, the capacity of the channel (22) is given as

$$
C_{\mathrm{CSIR}}\left(\tau, \gamma_{\tau}, \boldsymbol{X}_{1: \tau}\right)=-\frac{N(\ell-\tau)}{\ell} \log _{2}(e)-\frac{1}{m \ell} \kappa\left(\mathrm{u} \mid \mathbf{X}_{1}, \mathbf{z}, \hat{\mathbf{h}}\right),
$$

where the random variable $\mathrm{u}$ is defined as in (30) with independent random vectors $\mathbf{z} \sim \mathcal{C N}\left(\mathbf{0}, \boldsymbol{I}_{N m(\ell-\tau)}\right)$ and $\hat{\mathbf{h}} \sim$ $\mathcal{C N}\left(\mathbf{0}, \boldsymbol{I}_{N K}-\boldsymbol{\Gamma}_{\mathrm{MMSE}}\right)$, and independent random matrices $\mathbf{X}_{1}, \mathbf{X}_{2} \sim p_{\mathbf{X}}(\boldsymbol{X})=1 /|\mathcal{C}|^{\ell-\tau}$ for all $\boldsymbol{X} \in \mathcal{C}^{1 \times(\ell-\tau)}$. Furthermore, for $\tau \geq K$, we have the high-SNR limit

$$
\lim _{P \rightarrow \infty} C_{\mathrm{CSIR}}\left(\tau, \gamma_{\tau}, \boldsymbol{X}_{1: \tau}\right)=\frac{(\ell-\tau) \log _{2}(|\mathcal{C}|)}{m \ell} .
$$

Proof: It follows from the proof of Proposition 1 with the caveat that, since the channel estimate $\hat{\mathbf{h}}$ is available only at the receiver, the optimal input distribution $p_{\mathbf{X}}(\boldsymbol{X})$ is uniform. This is because the channel coefficients in vector $\overline{\mathbf{h}}(10)$ have uniformly distributed phases (see [31, Sec. VII]).

Prior works [8]-[14] have considered RIS-based modulation schemes that modulate the RIS reflection pattern independently from the transmitted symbols. By Corollary 1, an RISbased modulation scheme with independent and uniformly generated random codebooks for the transmitted symbols and reflection pattern is optimal when the transmitter has no access to CSI, and hence it cannot use the RIS for beamforming. Furthermore, since the high-SNR limits in Proposition 1 and 
Corollary 1 are equal, the availability of the CSI at the transmitter does not increase the capacity in the high-SNR regime.

Corollary 2 [23, Proposition 1]: When perfect CSI is available at both the receiver and transmitter, the capacity of the channel (22) is given as

$$
C_{\text {perfect }}=-N \log _{2}(e)-\min _{\substack{p_{\overline{\mathbf{x}}}(\overline{\mathbf{h}}|\overline{\mathbf{h}}| \overline{\mathbf{h}}): \\ \mathbb{E}\left[\operatorname{tr}\left(\overline{\mathbf{X}} \overline{\mathbf{X}} \overline{\mathbf{X}}^{*}\right)\right] \leq K m, \overline{\mathbf{X}} \in \mathcal{C}}} \frac{1}{m} \kappa\left(\tilde{\mathbf{u}} \mid \overline{\mathbf{X}}_{1}, \mathbf{z}, \overline{\mathbf{h}}\right),
$$

where the random variable $\tilde{u}$ is defined as

$$
\tilde{\mathbf{u}} \triangleq-\left\|\mathbf{z}+\gamma_{d}\left(\overline{\mathbf{X}}_{1}^{\otimes}-\overline{\mathbf{X}}_{2}^{\otimes}\right) \overline{\mathbf{h}}\right\|^{2}
$$

with independent random vectors $\mathbf{z} \sim \mathcal{C N}\left(\mathbf{0}, \boldsymbol{I}_{N m}\right), \overline{\mathbf{h}} \sim$ $\mathcal{C N}\left(\mathbf{0}, \boldsymbol{I}_{N K}\right)$, and random matrices $\overline{\mathbf{X}}_{1}, \overline{\mathbf{X}}_{2} \sim p_{\overline{\mathbf{X}} \mid \overline{\mathbf{h}}}(\overline{\boldsymbol{X}} \mid \overline{\boldsymbol{h}})$ that are conditionally independent given $\overline{\mathbf{h}}$. Furthermore, we have the high-SNR $\operatorname{limit}_{\lim } \rightarrow \infty C_{\text {perfect }}=\log _{2}(|\mathcal{C}|) / m$.

Proof: See Appendix B.

As shown in Appendix B, for perfect CSI, i.e., for $\hat{\mathbf{h}}(t)=$ $\overline{\mathbf{h}}(t)$, i.i.d. random coding across sub-blocks is optimal, which is reflected in the optimization over the distribution $p_{\overline{\mathbf{X}} \mid \overline{\mathbf{h}}}(\overline{\boldsymbol{X}} \mid \overline{\boldsymbol{h}})$ with $\overline{\mathbf{X}} \in \mathcal{C}$ in (36). This is because, when both transmitter and receiver know the CSI $\overline{\mathbf{h}}(t)$, the channel in (11) can be viewed as $\ell$ parallel Gaussian channels, for which independent encoding of the channels' input and separate decoding of each output is optimal [32, Ch. 9.4].

\section{A. Max-SNR Approach}

Having observed that achieving the capacity generally requires joint encoding of data over the codeword symbols and the RIS reflection pattern, we now consider the standard approach in which the reflection pattern of the RIS is fixed for all data sub-blocks $i=\tau+1, \ldots, \ell$, of the fading block $t$, irrespective of the message $w$, i.e., $\boldsymbol{\theta}_{i}(t)=\boldsymbol{\theta}(t)$. We denote the fixed RIS reflection pattern by $\boldsymbol{\theta}(\hat{\boldsymbol{h}})$ to emphasize that it is chosen based on the channel estimate $\hat{h}$ to maximize the achievable rate, and we have the following result.

Proposition 2: When the MMSE estimate $\hat{\mathbf{h}}$ in (19) is available at both the receiver and transmitter, an encoding scheme that selects the phase shift vector $\theta(\hat{\boldsymbol{h}})$ as a function of $\hat{\boldsymbol{h}}$ achieves the rate

$$
\begin{aligned}
& R_{\max -\mathrm{SNR}}\left(\tau, \gamma_{\tau}, \boldsymbol{X}_{1: \tau}\right) \\
& =-\frac{N(\ell-\tau)}{\ell} \log _{2}(e) \\
& -\min _{\boldsymbol{\theta}(\hat{\boldsymbol{h}}):} \min _{p_{\mathbf{X} \mid \hat{\boldsymbol{h}}}(\boldsymbol{X} \mid \hat{\boldsymbol{h}}):} \frac{1}{m \ell} \kappa\left(\mathrm{u} \mid \mathbf{X}_{1}, \mathbf{z}, \hat{\mathbf{h}}\right), \\
& \boldsymbol{\theta}(\hat{\boldsymbol{h}}) \in \mathcal{A}^{K \times 1} \mathbb{E}\left[\operatorname{tr}\left(\mathbf{X X}^{*}\right)\right] \leq K m(\ell-\tau), \\
& \mathbf{X} \in \mathcal{C}(\boldsymbol{\theta}(\hat{\boldsymbol{h}}))^{1 \times(\ell-\tau)}
\end{aligned}
$$

where the random variable $\mathrm{u}$ is defined as in (30) with independent random vectors $\mathbf{z} \sim \mathcal{C N}\left(\mathbf{0}, \boldsymbol{I}_{N m(\ell-\tau)}\right), \hat{\mathbf{h}} \sim$ $\mathcal{C N}\left(\mathbf{0}, \boldsymbol{I}_{N K}-\boldsymbol{\Gamma}_{\text {MMSE }}\right)$, and random matrices $\mathbf{X}_{1}, \mathbf{X}_{2} \sim$ $p_{\mathbf{X} \mid \hat{\mathbf{h}}}(\boldsymbol{X} \mid \hat{\boldsymbol{h}})$ that are conditionally independent given $\hat{\mathbf{h}}$. Furthermore, for $\tau \geq 1$, we have the high-SNR limit

$$
\lim _{P \rightarrow \infty} R_{\max -\mathrm{SNR}}\left(\tau, \gamma_{\tau}, \boldsymbol{X}_{1: \tau}\right)=\frac{(\ell-\tau) \log _{2}(S)}{\ell} .
$$

Proof: For a fixed RIS reflection pattern $\boldsymbol{\theta}_{i}(t)=\boldsymbol{\theta}(\hat{\boldsymbol{h}}(t))$ with $i=\tau+1, \ldots, \ell$, the channel input $\mathbf{X}(t)$ in (22) is restricted to the finite set $\mathcal{C}(\boldsymbol{\theta}(\hat{\boldsymbol{h}}(t)))^{1 \times(\ell-\tau)}$ in (9). Therefore, the result follows from Proposition 1 by restricting the input such that only the codeword symbols vary over the data subblocks. In (38), this is reflected in the optimization over the distribution $p_{\mathbf{X} \mid \hat{\mathbf{h}}}(\boldsymbol{X} \mid \hat{\boldsymbol{h}})$ with $\mathbf{X} \in \mathcal{C}(\boldsymbol{\theta}(\hat{\boldsymbol{h}}))^{1 \times(\ell-\tau)}$, where the RIS reflection pattern $\boldsymbol{\theta}(\hat{\boldsymbol{h}})$ is fixed. In addition, the limit (39) follows from (31) since, for any fixed RIS reflection pattern $\boldsymbol{\theta}(\hat{\boldsymbol{h}})$, we have $|\mathcal{C}(\boldsymbol{\theta}(\hat{\boldsymbol{h}}))|=S^{m}$.

The limit in (39) implies that, in the high-SNR regime, the rate of the max-SNR scheme is limited to $(\ell-\tau) \log _{2}(S) / \ell$. This is because, in each coherence block, the information data is modulated solely onto the $m(\ell-\tau)$ codeword symbols, which are selected from a constellation $\mathcal{S}$ of $S$ points. By comparing (39) with (31), we evince that, for any phase response set $\mathcal{A}$ of $A$ distinct phases, modulating the RIS reflection pattern can be used to increase the achievable rate by additional $K(\ell-\tau) \log _{2}(A) /(m \ell)$ bits per symbol as compared to the max-SNR scheme. However, note that the max-SNR scheme can achieve the high-SNR rate (39) by fixing the RIS reflection pattern irrespective of the CSI and estimating only the effective channel from the transmitter to the receiver. Therefore, the max-SNR approach requires only $\tau \geq 1$ pilot symbols to achieve the high-SNR limit in (39), whereas joint encoding achieves the limit in (31) with $\tau \geq K$ pilot symbols. For finite values of the SNR, the achievable rate in (38) can be computed by combining convex optimization tools for the inner minimization problem and global optimization tools for the minimization over the set of discrete phase shifts. The corresponding performance loss is evaluated in Section VI via numerical experiments.

The rates achieved for imperfect CSI available only at the receiver and for perfect CSI available at both the transmitter and receiver are given in the following two corollaries, respectively.

Corollary 3: When the MMSE estimate $\hat{\mathbf{h}}$ in (19) is available only at the receiver, a transmission scheme in which the phase shift vector $\boldsymbol{\theta}$ is kept fixed achieves the rate

$$
\begin{aligned}
R_{\max -S N R}^{\operatorname{CSIR}}\left(\tau, \gamma_{\tau}, \boldsymbol{X}_{1: \tau}\right)= & -\frac{N(\ell-\tau)}{\ell} \log _{2}(e) \\
& -\min _{\boldsymbol{\theta}: \boldsymbol{\theta} \in \mathcal{A}^{K \times 1}} \frac{1}{m \ell} \kappa\left(\mathrm{u} \mid \mathbf{X}_{1}, \mathbf{z}, \hat{\mathbf{h}}\right),
\end{aligned}
$$

where the random variable $\mathrm{u}$ is defined as in (30) with independent random vectors $\mathbf{z} \sim \mathcal{C N}\left(\mathbf{0}, \boldsymbol{I}_{N m(\ell-\tau)}\right)$ and $\hat{\mathbf{h}} \sim$ $\mathcal{C N}\left(\mathbf{0}, \boldsymbol{I}_{N K}-\boldsymbol{\Gamma}_{\mathrm{MMSE}}\right)$, and independent random matrices $\mathbf{X}_{1}, \mathbf{X}_{2} \sim p_{\mathbf{X}}(\boldsymbol{X})=1 /|\mathcal{C}(\boldsymbol{\theta})|^{\ell-\tau}$ for all $\boldsymbol{X} \in \mathcal{C}(\boldsymbol{\theta})^{1 \times(\ell-\tau)}$. Furthermore, for $\tau \geq 1$, we have the high-SNR limit

$$
\lim _{P \rightarrow \infty} R_{\max -\mathrm{SNR}}^{\mathrm{CSIR}}\left(\tau, \gamma_{\tau}, \boldsymbol{X}_{1: \tau}\right)=\frac{(\ell-\tau) \log _{2}(S)}{\ell} .
$$

Proof: It follows from the proof of Proposition 2 with the caveat that, since the channel estimate $\hat{\mathbf{h}}$ is available only at the receiver, the optimal input distribution $p_{\mathbf{X}}(\boldsymbol{X})$ is uniform. This is because the channel coefficients in vector $\overline{\mathbf{h}}(10)$ have uniformly distributed phases (see [31, Sec. VII]).

Corollary 4 [23, Proposition 2]: When the CSI is perfectly available at both the receiver and transmitter, a transmission scheme that selects the phase shift vector $\boldsymbol{\theta}(\overline{\boldsymbol{h}})$ as a function 
of $\overline{\boldsymbol{h}}$ achieves the rate

$$
\begin{aligned}
& R_{\text {max-SNR }}^{\text {perfect }}=-N \log _{2}(e)
\end{aligned}
$$

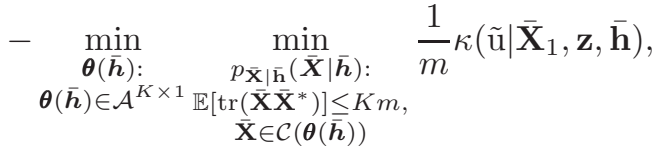

where the random variable $\tilde{\mathrm{u}}$ is defined as in (37) with independent random vectors $\mathbf{z} \sim \mathcal{C N}\left(\mathbf{0}, \boldsymbol{I}_{N m}\right), \overline{\mathbf{h}} \sim \mathcal{C N}\left(\mathbf{0}, \boldsymbol{I}_{N K}\right)$, and random matrices $\overline{\mathbf{X}}_{1}, \overline{\mathbf{X}}_{2} \sim p_{\overline{\mathbf{X}} \mid \overline{\mathbf{h}}}(\overline{\boldsymbol{X}} \mid \overline{\boldsymbol{h}})$ that are conditionally independent given $\overline{\mathbf{h}}$. Furthermore, we have the highSNR limit $\lim _{P \rightarrow \infty} R_{\text {max-SNR }}^{\text {perfect }}=\log _{2}(S)$.

Proof: Similar to the proof of Proposition 2, it follows from Corollary 2 by restricting the input such that only the codeword symbols vary over the data sub-blocks, whereas the RIS reflection is fixed.

\section{LAYERED ENCODING}

As discussed, achieving the capacity in (29) requires jointly encoding the message over the phase shift vector $\boldsymbol{\theta}_{i}(t)$ and the transmitted signal $\mathbf{s}_{i}(t)$, while performing optimal, i.e., maximum-likelihood joint decoding at the receiver. This may be infeasible in some communication networks. Therefore, in this section, we propose a strategy based on layered encoding and successive cancellation decoding (SCD) that uses only standard separate encoding and decoding procedures, while still benefiting from the modulation of information onto the state of the RIS so as to enhance the achievable rate compared with the max-SNR scheme.

To this end, the message $w$ is split into two sub-messages, or layers, $w_{1}$ and $w_{2}$, such that $w_{1}$, of rate $R_{1}$, is encoded onto the phase shift vectors $\boldsymbol{\theta}_{i}(t) \in \mathcal{A}^{K}$, whereas $w_{2}$, of rate $R_{2}$, is encoded onto the transmitted signals $\mathbf{s}_{i}(t)=$ $\left(\mathrm{s}_{i, 1}(t), \ldots, \mathrm{s}_{i, m}(t)\right)^{\top}$, for $i=\tau+1, \ldots, \ell$ and $t \in[n / T]$. In order to enable decoding using standard SCD, the first $\mu \geq 1$ symbols in the vectors $\mathbf{s}_{i}(t)$ are fixed and used as additional pilot symbols. In particular, we have

$$
\mathrm{s}_{i, q}(t) \equiv 1, \quad i=\tau+1, \ldots, \ell, q \in[\mu], t \in[n / T] .
$$

It is worth clarifying that the pilot symbols discussed in Section II-A are employed for channel estimation, while the additional pilot symbols introduced in this section facilitate the separate decoding of the two layers, as detailed next. The pilot symbols in (43) are necessary because the channel estimation pilot symbols cannot be used for SCD since both the transmitted symbols and RIS reflection pattern are fixed during the channel estimation phase.

By averaging the first $\mu$ columns of the received signal matrix $\mathbf{Y}_{i}(t)$ in $(7)$, we obtain

$$
\overline{\mathbf{y}}_{i}(t) \triangleq \frac{1}{\sqrt{\mu}} \sum_{q=1}^{\mu} \mathbf{y}_{i, q}(t)=\sqrt{\mu} \gamma_{d} \mathbf{H}(t) e^{j \boldsymbol{\theta}_{i}(t)}+\overline{\mathbf{z}}_{i}(t),
$$

where we have defined random vector $\overline{\mathbf{z}}_{i}(t) \sim \mathcal{C N}\left(\mathbf{0}, \boldsymbol{I}_{N}\right)$. The receiver decodes layer $w_{1}$ based on the received matrix $\overline{\mathbf{Y}}(t) \triangleq\left(\overline{\mathbf{y}}_{\tau+1}(t), \ldots, \overline{\mathbf{y}}_{\ell}(t)\right)$, which, from (44), can be expressed as

$$
\overline{\mathbf{Y}}(t)=\gamma_{d} \mathbf{H}(t) \mathbf{Q}(t)+\overline{\mathbf{Z}}(t)
$$

where we have defined the matrix $\overline{\mathbf{Z}}(t) \triangleq$ $\left(\overline{\mathbf{z}}_{\tau+1}(t), \ldots, \overline{\mathbf{z}}_{\ell}(t)\right) \in \mathbb{C}^{N \times(\ell-\tau)}$, whose elements are i.i.d. with distribution $\mathcal{C N}(0,1)$, and the phase shift matrix

$$
\mathbf{Q}(t) \triangleq\left(\begin{array}{ccc}
\sqrt{\mu} e^{j \theta_{\tau+1,1}(t)} & \cdots & \sqrt{\mu} e^{j \theta_{\ell, 1}(t)} \\
\vdots & \ddots & \vdots \\
\sqrt{\mu} e^{j \theta_{\tau+1, K}(t)} & \cdots & \sqrt{\mu} e^{j \theta_{\ell, K}(t)}
\end{array}\right),
$$

which is selected from the set

$$
\begin{gathered}
\mathcal{Q}(\ell-\tau) \triangleq\left\{\boldsymbol{Q} \in \mathbb{C}^{K \times(\ell-\tau)}: Q_{k, i}=\sqrt{\mu} e^{j \theta_{i, k}}, \theta_{i, k} \in \mathcal{A},\right. \\
k \in[K], i=\tau+1, \ldots, \ell\} .
\end{gathered}
$$

By direct inspection of (45), we evince that it depends only of the RIS phase shifts, and hence layer $w_{1}$ can be separately decoded. Once layer $w_{1}$ is decoded, the receiver reconstructs the phase shift vectors $\boldsymbol{\theta}_{i}(t)$, which are then used to decode layer $w_{2}$. This strategy achieves the rate detailed in Proposition 3.

Proposition 3: A strategy based on layered encoding and SCD achieves the rate

$$
\begin{aligned}
R_{\text {layered }}\left(\tau, \gamma_{\tau}, \boldsymbol{X}_{1: \tau}, \mu\right)= & R_{1}\left(\tau, \gamma_{\tau}, \boldsymbol{X}_{1: \tau}, \mu\right) \\
& +R_{2}\left(\tau, \gamma_{\tau}, \boldsymbol{X}_{1: \tau}, \mu\right),
\end{aligned}
$$

where the rate $R_{1}\left(\tau, \gamma_{\tau}, \boldsymbol{X}_{1: \tau}, \mu\right)$ is defined as

$$
\begin{aligned}
R_{1}\left(\tau, \gamma_{\tau}, \boldsymbol{X}_{1: \tau}, \mu\right)= & -\frac{N(\ell-\tau)}{m \ell} \log _{2}(e) \\
& -\frac{1}{m \ell} \kappa\left(\mathrm{u}_{1} \mid \mathbf{Q}_{1}, \overline{\mathbf{z}}, \hat{\mathbf{h}}\right)
\end{aligned}
$$

with the random variable $\mathrm{u}_{1}$

$\mathrm{u}_{1} \triangleq \ln \left(\frac{\left|\boldsymbol{\Gamma}\left(\mathbf{Q}_{1}\right)\right|}{\left|\boldsymbol{\Gamma}\left(\mathbf{Q}_{2}\right)\right|}\right)-\left\|\boldsymbol{V}\left(\mathbf{Q}_{1}\right) \overline{\mathbf{z}}+\gamma_{d}\left(\mathbf{Q}_{1}^{\otimes}-\mathbf{Q}_{2}^{\otimes}\right) \hat{\mathbf{h}}\right\|_{\boldsymbol{\Gamma}\left(\mathbf{Q}_{2}\right)}^{2}$

defined by independent random vectors $\overline{\mathbf{z}} \sim \mathcal{C N}\left(\mathbf{0}, \boldsymbol{I}_{N(\ell-\tau)}\right)$ and $\hat{\mathbf{h}} \sim \mathcal{C N}\left(\mathbf{0}, \boldsymbol{I}_{N K}-\boldsymbol{\Gamma}_{\mathrm{MMSE}}\right)$, and independent random matrices $\mathbf{Q}_{1}, \mathbf{Q}_{2} \sim p_{\mathbf{Q}}(\boldsymbol{Q})=1 / A^{K(\ell-\tau)}$ for all $\boldsymbol{Q} \in \mathcal{Q}(\ell-\tau)$; and where the rate $R_{2}\left(\tau, \gamma_{\tau}, \boldsymbol{X}_{1: \tau}, \mu\right)$ is defined as

$$
\begin{aligned}
R_{2}\left(\tau, \gamma_{\tau}, \boldsymbol{X}_{1: \tau}, \mu\right)= & -\frac{N(m-\mu)(\ell-\tau)}{m \ell} \log _{2}(e) \\
& -\frac{1}{m \ell} \kappa\left(\mathrm{u}_{2} \mid \check{\mathbf{X}}_{1}, \check{\mathbf{z}}, \hat{\mathbf{h}}, \boldsymbol{\theta}_{\tau+1}, \ldots, \boldsymbol{\theta}_{\ell}\right)
\end{aligned}
$$

with the random variable $\mathrm{u}_{2}$

$$
\mathrm{u}_{2} \triangleq \ln \left(\frac{\left|\boldsymbol{\Gamma}\left(\check{\mathbf{X}}_{1}\right)\right|}{\left|\boldsymbol{\Gamma}\left(\check{\mathbf{X}}_{2}\right)\right|}\right)-\left\|\boldsymbol{V}\left(\check{\mathbf{X}}_{1}\right) \check{\mathbf{z}}+\gamma_{d}\left(\check{\mathbf{X}}_{1}^{\otimes}-\check{\mathbf{X}}_{2}^{\otimes}\right) \hat{\mathbf{h}}\right\|_{\boldsymbol{\Gamma}\left(\check{\mathbf{X}}_{2}\right)}^{2}
$$

defined by independent random vectors $\check{\mathbf{z}} \sim$ $\mathcal{C N}\left(\mathbf{0}, \boldsymbol{I}_{N(m-\mu)(\ell-\tau)}\right), \quad \hat{\mathbf{h}} \sim \mathcal{C N}\left(\mathbf{0}, \boldsymbol{I}_{N K}-\boldsymbol{\Gamma}_{\mathrm{MMSE}}\right)$, $\boldsymbol{\theta}_{\tau+1}, \ldots, \boldsymbol{\theta}_{\ell} \sim p_{\boldsymbol{\theta}}(\boldsymbol{\theta})=1 / A^{K}$ for all $\boldsymbol{\theta} \in \mathcal{A}^{K}$, and independent random matrices $\check{\mathbf{X}}_{1}, \check{\mathbf{X}}_{2} \sim$ $p_{\check{\mathbf{X}} \mid \boldsymbol{\theta}_{\tau+1}, \ldots, \boldsymbol{\theta}_{\ell}}\left(\check{\boldsymbol{X}} \mid \boldsymbol{\theta}_{\tau+1}, \ldots, \boldsymbol{\theta}_{\ell}\right)=1 / S^{(m-\mu)(\ell-\tau)}$ for all $\check{\boldsymbol{X}} \in \mathcal{C}\left(\boldsymbol{\theta}_{\tau+1}, \ldots, \boldsymbol{\theta}_{\ell} ; \mu\right)$ with

$$
\begin{array}{r}
\mathcal{C}\left(\boldsymbol{\theta}_{\tau+1}, \ldots, \boldsymbol{\theta}_{\ell} ; \mu\right) \triangleq\left\{\check{\boldsymbol{X}}: \check{\boldsymbol{X}}=\left(e^{j \boldsymbol{\theta}_{\tau+1}} \check{\mathbf{s}}_{\tau+1}^{\top}, \ldots, e^{j \boldsymbol{\theta}_{\ell}} \check{\mathbf{s}}_{\ell}^{\top}\right),\right. \\
\left.\check{\mathbf{s}}_{i} \in \mathcal{S}^{(m-\mu) \times 1}, i=\tau+1, \ldots, \ell\right\} .
\end{array}
$$


Furthermore, for $\tau \geq K$, we obtain the high-SNR limit

$$
\begin{aligned}
\lim _{P \rightarrow \infty} R_{\text {layered }}\left(\tau, \gamma_{\tau}, \boldsymbol{X}_{1: \tau}, \mu\right) \\
=\frac{\ell-\tau}{m \ell}\left[(m-\mu) \log _{2}(S)+K \log _{2}(A)\right] .
\end{aligned}
$$

Proof: See Appendix C.

Note that the layered encoding scheme does not require CSI at the transmitter (CSIT) since both layers are encoded independently from the channel estimate $\hat{\mathbf{h}}$. In addition, while the rate of the first layer increases with the number of additional pilots $\mu$ due to the averaging in (44), the rate of the second layer decreases as $\mu$ increases since fewer symbols at each sub-block can be used to encode sub-message $w_{2}$. Therefore, in order to maximize the achievable rate in (48), the number of pilots $\mu$ should be optimized. However, for sufficiently high SNR, the limit in (54) implies that setting $\mu=1$ maximizes the achievable rate. The rate achieved by the proposed layered strategy in the case of perfect CSI is derived in the following corollary.

Corollary 5 [23, Proposition 4]: Under the assumption that perfect CSI is available at the receiver, a strategy based on layered encoding and SCD achieves the rate

$$
R_{\text {layered }}^{\text {perfect }}(\mu)=R_{1}^{\text {perfect }}(\mu)+R_{2}^{\text {perfect }}(\mu),
$$

where the rate $R_{1}^{\text {perfect }}(\mu)$ is defined as

$$
R_{1}^{\text {perfect }}(\mu)=-\frac{N}{m} \log _{2}(e)-\frac{1}{m} \kappa\left(\tilde{\mathbf{u}}_{1} \mid \mathbf{Q}_{1}, \overline{\mathbf{z}}, \overline{\mathbf{h}}\right)
$$

with the random variable $\mathrm{u}_{1}$

$$
\tilde{\mathrm{u}}_{1} \triangleq-\left\|\overline{\mathbf{z}}+\gamma_{d}\left(\mathbf{Q}_{1}^{\otimes}-\mathbf{Q}_{2}^{\otimes}\right) \overline{\mathbf{h}}\right\|^{2}
$$

defined by independent random vectors $\overline{\mathbf{z}} \sim \mathcal{C N}\left(\mathbf{0}, \boldsymbol{I}_{N}\right)$ and $\overline{\mathbf{h}} \sim \mathcal{C N}\left(\mathbf{0}, \boldsymbol{I}_{N K}\right)$, and independent random matrices $\mathbf{Q}_{1}, \mathbf{Q}_{2} \sim p_{\mathbf{Q}}(\boldsymbol{Q})=1 / A^{K}$ for all $\boldsymbol{Q} \in \mathcal{Q}(1)$ (47); and where the rate $R_{2}^{\text {perfect }}(\mu)$ is defined as

$$
R_{2}^{\text {perfect }}(\mu)=-\frac{N(m-\mu)}{m} \log _{2}(e)-\frac{1}{m} \kappa\left(\tilde{\mathrm{u}}_{2} \mid \check{\mathbf{X}}_{1}, \check{\mathbf{z}}, \overline{\mathbf{h}}, \boldsymbol{\theta}\right)
$$

with the random variable $\mathrm{u}_{2}$

$$
\tilde{\mathrm{u}}_{2} \triangleq-\left\|\check{\mathbf{z}}+\gamma_{d}\left(\check{\mathbf{X}}_{1}^{\otimes}-\check{\mathbf{X}}_{2}^{\otimes}\right) \overline{\mathbf{h}}\right\|^{2}
$$

defined by independent random vectors $\check{\mathbf{z}} \sim \mathcal{C N}\left(\mathbf{0}, \boldsymbol{I}_{N(m-\mu)}\right)$, $\overline{\mathbf{h}} \sim \mathcal{C N}\left(\mathbf{0}, \boldsymbol{I}_{N K}\right), \boldsymbol{\theta} \sim p_{\boldsymbol{\theta}}(\boldsymbol{\theta})=1 / A^{K}$ for all $\boldsymbol{\theta} \in \mathcal{A}^{K}$, and independent random matrices $\check{\mathbf{X}}_{1}, \check{\mathbf{X}}_{2} \sim p_{\check{\mathbf{X}} \mid \boldsymbol{\theta}}(\check{\boldsymbol{X}} \mid \boldsymbol{\theta})=$ $1 / S^{(m-\mu)}$ for all $\check{\boldsymbol{X}} \in \mathcal{C}(\boldsymbol{\theta} ; \mu)(53)$.

Proof: Similar to the proof of Corollary 2, it follows from the proof of Proposition 3 by noting that, for perfect CSI, i.e., $\hat{\mathbf{h}}(t)=\overline{\mathbf{h}}(t)$ and $\tau=0$, separate decoding of the subblocks is optimal.

\section{LOWER BOUNDS}

As discussed in the previous sections, calculating the capacity and achievable rates typically requires the evaluation of expectations over Gaussian random vectors and over discrete random matrices whose size increases exponentially with $\ell-\tau$. This makes the evaluation numerically difficult for long coherence blocks. Furthermore, unlike the Gaussian vectors that have a known distribution, the input distribution of the random matrices needs to be numerically optimized. This implies that the standard method for estimating the expectations via empirical averages cannot be applied to the discrete random matrices, and hence estimating the expectations from a small number of samples requires methods such as the Monte Carlo gradient estimation [33]. In this section, we take a different approach and present lower bounds on the capacity and achievable rates that require summing over a fixed number of terms that does not increase with the number of sub-blocks $\ell$, which simplifies the exact calculation of the bounds.

\section{A. Lower Bounds for Optimal Signalling and Max-SNR}

Proposition 4: When the MMSE estimate $\hat{\mathbf{h}}$ in (19) is available at both the receiver and transmitter, the capacity in Proposition 1 and the rate achieved by the max-SNR scheme in Proposition 2 are lower bounded as $C\left(\tau, \gamma_{\tau}, \boldsymbol{X}_{1: \tau}\right) \geq \underline{C}\left(\tau, \gamma_{\tau}, \boldsymbol{X}_{1: \tau}\right)$ and $R_{\max -\mathrm{SNR}}\left(\tau, \gamma_{\tau}, \boldsymbol{X}_{1: \tau}\right) \geq \underline{R}_{\max -\mathrm{SNR}}\left(\tau, \gamma_{\tau}, \boldsymbol{X}_{1: \tau}\right)$, respectively, where

$$
\begin{aligned}
C\left(\tau, \gamma_{\tau}, \boldsymbol{X}_{1: \tau}\right) \triangleq & -\frac{N(\ell-\tau)}{\ell} \log _{2}(e) \\
& -\min _{\substack{p_{\overline{\mathbf{x}} \mid \hat{h}}(\overline{\mathbf{X}} \mid \hat{\boldsymbol{h}}): \\
\mathbb{E}\left[\operatorname{tr}\left(\overline{\mathbf{X}}^{*} \overline{\mathbf{X}}^{*}\right)\right] \leq K m, \overline{\mathbf{X}} \in \mathcal{C}}} \frac{\ell-\tau}{m \ell} \kappa\left(\mathrm{u} \mid \overline{\mathbf{X}}_{1}, \mathbf{z}, \hat{\mathbf{h}}\right),
\end{aligned}
$$

and

$$
\begin{aligned}
& \underline{R}_{\max -\mathrm{SNR}}\left(\tau, \gamma_{\tau}, \boldsymbol{X}_{1: \tau}\right) \\
& \triangleq-\frac{N(\ell-\tau)}{\ell} \log _{2}(e) \\
& -\min _{\boldsymbol{\theta}(\hat{\boldsymbol{h}}):} \min _{p_{\overline{\mathbf{X}} \mid \hat{\mathbf{h}}(\overline{\mathbf{X}} \mid \hat{\boldsymbol{h}}):}} \frac{\ell-\tau}{m \ell} \kappa\left(\mathrm{u} \mid \overline{\mathbf{X}}_{1}, \mathbf{z}, \hat{\mathbf{h}}\right) . \\
& \boldsymbol{\theta}(\hat{\boldsymbol{h}}) \in \mathcal{A}^{K \times 1} \mathbb{E}\left[\operatorname{tr}\left(\overline{\mathbf{X}} \overline{\mathbf{X}}^{*}\right)\right] \leq K m, \\
& \overline{\mathbf{X}} \in \mathcal{C}(\boldsymbol{\theta}(\hat{\boldsymbol{h}}))
\end{aligned}
$$

The random variable $\mathrm{u}$ in (60) and (61) is defined as in (30) with independent random vectors $\mathbf{z} \sim \mathcal{C N}\left(\mathbf{0}, \boldsymbol{I}_{N m}\right)$, $\hat{\mathbf{h}} \sim \mathcal{C N}\left(\mathbf{0}, \boldsymbol{I}_{N K}-\boldsymbol{\Gamma}_{\mathrm{MMSE}}\right)$, and random matrices $\overline{\mathbf{X}}_{1}, \overline{\mathbf{X}}_{2} \sim$ $p_{\overline{\mathbf{X}} \mid \hat{\mathbf{h}}}(\overline{\boldsymbol{X}} \mid \hat{\boldsymbol{h}})$ that are conditionally independent given $\hat{\mathbf{h}}$.

Proof: See Appendix D.

As detailed in Appendix D, the lower bounds in Proposition 4 correspond to rates achievable when the subblocks $\overline{\mathbf{X}}_{i} \in \mathcal{C}, i=\tau+1, \ldots, \ell$, are encoded and decoded separately. This is in contrast to the optimal strategy presented in Proposition 1 that jointly decodes all data subblocks inputs $\left(\overline{\mathbf{X}}_{\tau+1}, \ldots, \overline{\mathbf{X}}_{\ell}\right) \in \mathcal{C}^{\ell-\tau}$ from the channel outputs $\mathbf{y}_{\tau+1}, \ldots, \mathbf{y}_{\ell}$. The key computational advantage of the lower bounds is that evaluating the expectations over the discrete random matrices $\mathbf{X}_{1}$ and $\mathbf{X}_{2}$ defined in Proposition 1 requires summing over $|\mathcal{C}|^{\ell-\tau}$ terms, whereas evaluating the expectations in the lower bound (60) requires summing over $|\mathcal{C}|$ terms, which is exponentially smaller.

In addition, as shown in Appendix D, for perfect CSI, i.e., $\hat{\mathbf{h}}(t)=\overline{\mathbf{h}}(t)$ and $\tau=0$, the lower bounds in Proposition 4 coincide with the capacity and achievable rates in Corollary 2 and Corollary 4. This is because, for perfect channel estimation, independent encoding of the sub-blocks is optimal. 
Therefore, based on this result, the lower bounds are expected to be close to the capacity when channel estimation is accurate, e.g., for $\tau \geq K$ and for sufficiently high SNR, as demonstrated in Section VI via numerical experiments.

The corresponding lower bounds on capacity and rate achieved by the max-SNR scheme under the assumptions of imperfect CSI available only at the receiver are formulated in the following corollary.

Corollary 6: When the MMSE estimate $\hat{\mathbf{h}}$ in (19) is available only at the receiver, the capacity in Corollary 1 and the rate achieved by the max-SNR scheme in Corollary 3 are lower bounded as $C_{\mathrm{CSIR}}\left(\tau, \gamma_{\tau}, \boldsymbol{X}_{1: \tau}\right) \geq \underline{C}_{\mathrm{CSIR}}\left(\tau, \gamma_{\tau}, \boldsymbol{X}_{1: \tau}\right)$ and $R_{\max -\mathrm{SNR}}^{\mathrm{CSIR}}\left(\tau, \gamma_{\tau}, \boldsymbol{X}_{1: \tau}\right) \geq \underline{R}_{\max -\mathrm{SNR}}^{\mathrm{CSIR}}\left(\tau, \gamma_{\tau}, \boldsymbol{X}_{1: \tau}\right)$, respectively, where

$$
\begin{aligned}
\underline{C}_{\mathrm{CSIR}}\left(\tau, \gamma_{\tau}, \boldsymbol{X}_{1: \tau}\right) \triangleq & -\frac{N(\ell-\tau)}{\ell} \log _{2}(e) \\
& -\frac{\ell-\tau}{m \ell} \kappa\left(\mathrm{u} \mid \overline{\mathbf{X}}_{1}, \mathbf{z}, \hat{\mathbf{h}}\right)
\end{aligned}
$$

and

$$
\begin{aligned}
\underline{R}_{\max -S \mathrm{SNR}}^{\mathrm{CSIR}}\left(\tau, \gamma_{\tau}, \boldsymbol{X}_{1: \tau}\right) \triangleq & -\frac{N(\ell-\tau)}{\ell} \log _{2}(e) \\
& -\min _{\boldsymbol{\theta}: \boldsymbol{\theta} \in \mathcal{A}^{K \times 1}} \frac{\ell-\tau}{m \ell} \kappa\left(\mathrm{u} \mid \overline{\mathbf{X}}_{1}, \mathbf{z}, \hat{\mathbf{h}}\right) .
\end{aligned}
$$

The random variable $u$ in (62) and (63) is defined as in (30) with independent random vectors $\mathbf{z} \sim \mathcal{C N}\left(\mathbf{0}, \boldsymbol{I}_{N m}\right)$ and $\hat{\mathbf{h}} \sim \mathcal{C N}\left(\mathbf{0}, \boldsymbol{I}_{N K}-\boldsymbol{\Gamma}_{\mathrm{MMSE}}\right)$, and independent random matrices $\overline{\mathbf{X}}_{1}, \overline{\mathbf{X}}_{2} \sim p_{\overline{\mathbf{X}}}(\overline{\boldsymbol{X}})$, where $p_{\overline{\mathbf{X}}}(\overline{\boldsymbol{X}})=1 /|\mathcal{C}|$ in (62) and $p_{\overline{\mathbf{X}}}(\overline{\boldsymbol{X}})=1 /|\mathcal{C}(\boldsymbol{\theta})|$ in (63).

Proof: It follows from the proof of Proposition 4 with the caveat that, since the channel estimate $\hat{\mathbf{h}}$ is available only at the receiver, the optimal input distribution $p_{\mathbf{X}}(\boldsymbol{X})$ is uniform. This is because the channel coefficients in vector $\overline{\mathbf{h}}(10)$ have uniformly distributed phases (see [31, Sec. VII]).

\section{B. Lower Bound for Layered Encoding}

Similar to Proposition 4, we derive a lower bound on the rate achieved by the layered-encoding scheme introduced in Section IV.

Proposition 5: The achievable rate of the layered encoding scheme introduced in Section IV is lower bounded as $R_{\text {layered }}\left(\tau, \gamma_{\tau}, \boldsymbol{X}_{1: \tau}, \mu\right) \geq \underline{R}_{\text {layered }}\left(\tau, \gamma_{\tau}, \boldsymbol{X}_{1: \tau}, \mu\right)$ with

$$
\begin{aligned}
\underline{R}_{\text {layered }}\left(\tau, \gamma_{\tau}, \boldsymbol{X}_{1: \tau}, \mu\right) \triangleq-\frac{\ell-\tau}{m \ell} & {\left[N(m+1-\mu) \log _{2}(e)\right.} \\
& +\kappa\left(\mathrm{u}_{1} \mid \mathbf{Q}_{1}, \overline{\mathbf{z}}, \hat{\mathbf{h}}\right) \\
& \left.+\kappa\left(\mathrm{u}_{2} \mid \check{\mathbf{X}}_{1}, \check{\mathbf{z}}, \hat{\mathbf{h}}, \boldsymbol{\theta}\right)\right],
\end{aligned}
$$

where the random variable $u_{1}$ is defined as in (50) with independent random vectors $\overline{\mathbf{z}} \sim \mathcal{C N}\left(\mathbf{0}, \boldsymbol{I}_{N}\right)$ and $\hat{\mathbf{h}} \sim$ $\mathcal{C N}\left(\mathbf{0}, \boldsymbol{I}_{N K}-\boldsymbol{\Gamma}_{\mathrm{MMSE}}\right)$, and independent random matrices $\mathbf{Q}_{1}, \mathbf{Q}_{2} \sim p_{\mathbf{Q}}(\boldsymbol{Q})=1 / A^{K}$ for all $\boldsymbol{Q} \in \mathcal{Q}(1)$; and where the random variable $u_{2}$ is defined as in (52) with independent random vectors $\check{\mathbf{z}} \sim \mathcal{C N}\left(\mathbf{0}, \boldsymbol{I}_{N(m-\mu)}\right), \hat{\mathbf{h}} \sim \mathcal{C N}\left(\mathbf{0}, \boldsymbol{I}_{N K}-\right.$ $\left.\boldsymbol{\Gamma}_{\mathrm{MMSE}}\right), \boldsymbol{\theta} \sim p_{\boldsymbol{\theta}}(\boldsymbol{\theta})=1 / A^{K}$ for all $\boldsymbol{\theta} \in \mathcal{A}^{K}$, and independent random matrices $\mathbf{X}_{1}, \check{\mathbf{X}}_{2} \sim p_{\check{\mathbf{X}} \mid \boldsymbol{\theta}}(\check{\boldsymbol{X}} \mid \boldsymbol{\theta})=1 / S^{(m-\mu)}$ for all $\check{\boldsymbol{X}} \in\left\{\check{\boldsymbol{X}}: \check{\boldsymbol{X}}=e^{j \boldsymbol{\theta}} \check{\mathbf{s}}^{\top}, \check{\mathbf{s}} \in \mathcal{S}^{(m-\mu) \times 1}\right\}$.

Proof: See Appendix E.
Note that, similar to the capacity and rate achieved by the max-SNR scheme, the lower bound in Proposition 5 coincides with the achievable rate in Corollary 5 for perfect CSI.

\section{Numerical Results}

In this section, we illustrate and discuss numerical examples with the main aims of (i) comparing the capacity achieved by the proposed joint encoding scheme with the achievable rates attained by the max-SNR and the layered encoding schemes, and (ii) assessing the impact of imperfect CSI. For the phase response set, we consider $A$ uniformly spaced phases in the set $\mathcal{A} \triangleq\{0,2 \pi / A, \ldots, 2 \pi(A-1) / A\}$, whereas, for the input constellation, we consider ASK, which was shown to maximize the capacity in the high-SNR regime (Proposition 1), and PSK modulations. In addition, we set an equal power for training and data sub-blocks, i.e., $\gamma_{\tau}=\gamma_{d}=\sqrt{P}$, and optimize the channel estimation by testing all pilot symbols $\boldsymbol{X}_{1: \tau} \in \mathcal{C}^{1 \times \tau}$ that satisfy the power constraint in (14). Moreover, the empirical average over Gaussian random vectors, e.g., $\hat{\mathbf{h}}$ and $\mathbf{z}$ in Proposition 1, is evaluated via a Monte Carlo method, and the optimal input distributions, e.g., $p_{\mathbf{X} \mid \hat{\mathbf{h}}}(\boldsymbol{X} \mid \hat{\boldsymbol{h}})$ in Proposition 1, are numerically calculated using the fmincon function in MATLAB.

We limit our investigation to small number of RIS elements $K$ in order to numerically evaluate the capacity and lower bound without requiring excessive computing power for optimizing the input distribution in (29) and (60), respectively. It is noted that the complexity of optimizing the input distribution does not reflect the implementation complexity at run time. For instance, as discussed in Section III, in some regimes, uniform distributions are optimal, which can be realized with standard codes. More generally, when the distribution is not uniform, probability shaping methods can be leveraged [34]. Based on the high-SNR analysis in Proposition 1, we can conclude that the capacity increases linearly with the number of elements $K$ for sufficiently high SNR and a sufficiently long coherence block. We postpone the numerical analysis with larger $K$ to future works.

On the role of the SNR level. In Fig. 4, we plot the rate as a function of the average power $P$, with $\ell=4$ sub-blocks of which $\tau=2$ sub-blocks are used for channel estimation, $N=$ 2 receive antennas, $K=2$ RIS elements, $A=2$ available phase shifts, a symbol-to-RIS control rate $m=1$, and an input constellation given by the 4-ASK $\mathcal{S}=\{\sigma, 3 \sigma, 5 \sigma, 7 \sigma\}$ with $\sigma=1 / \sqrt{21}$. For very low SNR, i.e., less than $-20 \mathrm{~dB}$, it is observed that the max-SNR approach is close to being optimal, and hence, in this regime, encoding information in the RIS reflection pattern does not increase the rate. For larger SNR levels of practical interest, however, joint encoding provides a significant gain over the max-SNR scheme.

It is also observed that CSIT is unnecessary for very low or very high SNR levels. This is because, at low SNR, the channel estimate is poor and cannot be applied for beamforming, whereas, at high SNR, beamforming, which is used to increase SNR, is unnecessary. In addition, the lower bounds presented in Section V are shown to be close to the achievable rates.

Optimal number of pilot symbols. In Fig. 5, we plot the lower bounds on the rate as a function of the number of training sub-blocks $\tau$ with $\ell=20$ sub-blocks in each coherence block, $N=2$ receive antennas, $K=4$ RIS elements, 


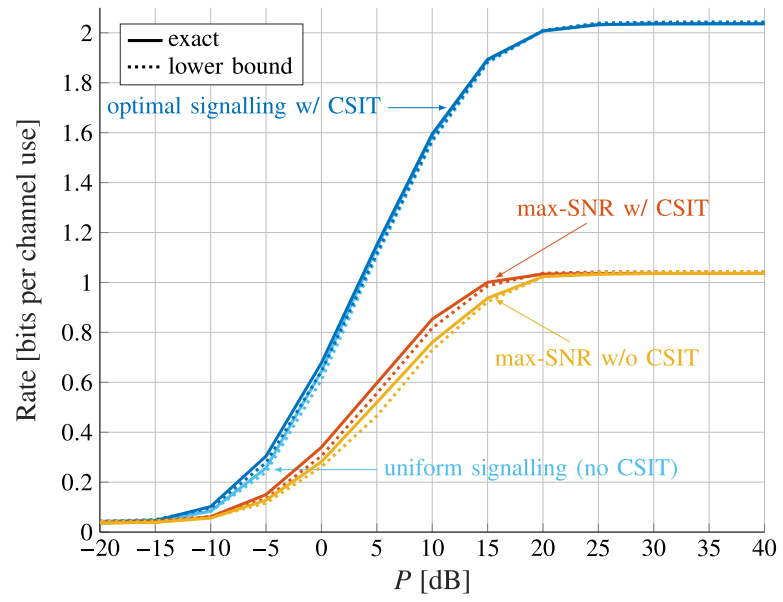

Fig. 4. Rates as a function of the normalized power $P[\mathrm{~dB}]$ for $\ell=4$, $\tau=2, N=2, K=2, A=2, m=1$, and 4-ASK input constellation.

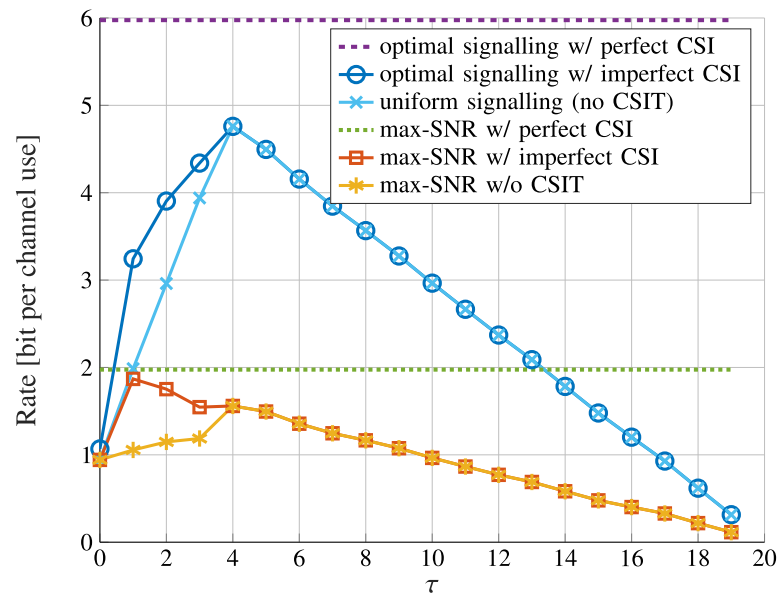

Fig. 5. Rate lower bounds as a function of the number of training sub-blocks $\tau$ for $\ell=20, N=2, K=4, A=2, m=1, P=40 \mathrm{~dB}$, and 4-ASK input constellation.

$A=2$ available phase shifts, a symbol-to-RIS control rate $m=1$, an average power constraint of $P=40 \mathrm{~dB}$, and an input constellation given by 4 -ASK. Note that we plot the lower bounds and not the exact expressions since evaluating the capacity requires summing over the set of channel inputs $\mathbf{X}$ whose size is $|\mathcal{C}|^{\ell-\tau}=\left(A^{K} \cdot S^{m}\right)^{\ell-\tau}=2^{120-6 \tau}$, which is not feasible. It is observed that the lower bound on the capacity increases with $\tau$ up to $\tau=4$, and then decreases. This is because increasing the number of pilot symbols improves the channel estimation accuracy on the one hand, but on the other hand leaves fewer sub-blocks for transmitting data. In addition, joint encoding is shown to require a more accurate channel estimation compared to the max-SNR scheme with CSIT, for which allocating $\tau=1$ pilot is optimal. Comparing the penalty of channel estimation between the joint encoding strategy and the max-SNR scheme, we observe that the gap is larger for joint encoding since a higher percentage of the coherence block is used to obtain a sufficient channel estimation accuracy.

As seen in Fig. 5, the capacity-achieving joint encoding strategy requires a better channel estimation compared to the max-SNR scheme. However, for short coherence blocks,

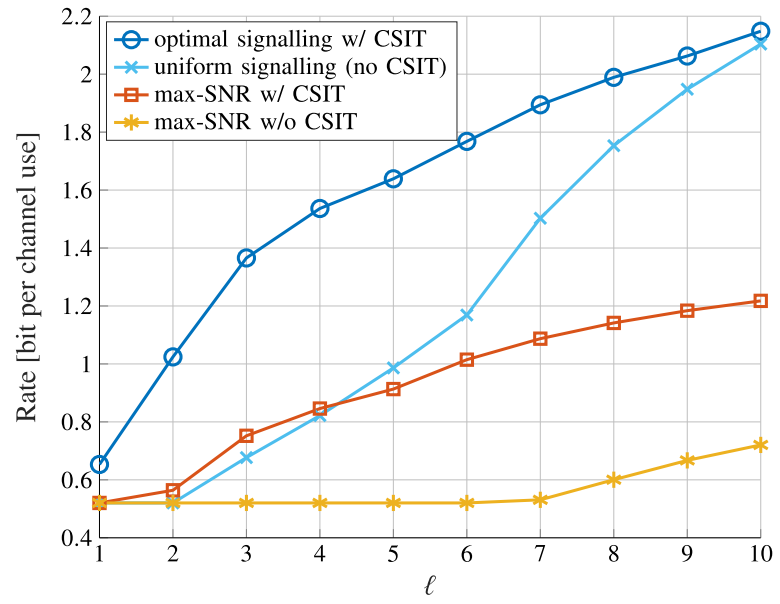

Fig. 6. Rate lower bounds as a function of the number of sub-blocks $\ell$ for $N=1, K=4, A=2, m=1, P=10 \mathrm{~dB}$, and 4-ASK input constellation.

acquiring sufficiently good channel estimation might not be feasible and the gain of joint encoding is expected to decrease. This is illustrated in Fig. 6, where we plot the lower bounds on the rate as a function of the number of sub-blocks $\ell$ with $N=2$ receive antennas, $K=4$ RIS elements, $A=2$ available phase shifts, a symbol-to-RIS control rate $m=1$, an average power constraint of $P=10 \mathrm{~dB}$, and an input constellation given by 4 -ASK. For each value of $\ell$, the lower bounds are optimized over $\tau=0, \ldots, \ell-1$. For fast-changing channels, the gain of joint encoding is shown to be low. Moreover, without CSIT, the max-SNR scheme is optimal for $\ell \leq 2$. In addition, Fig. 6 demonstrates the dependence of the optimal input distribution on the channel estimation quality. Specifically, for $\ell=1$ and $\ell=10$, i.e., when very poor or very good channel estimation can be obtained with minor overhead relative to the coherence block, uniform signalling is shown to achieve a close-to-optimal rate, whereas, for intermediate coherence blocks, joint encoding over the transmitted signal and RIS reflection pattern is required to achieve the capacity.

On the number of receive antennas. In Fig. 7, we plot the lower bounds on the rate as a function of the number of receive antennas $N$ with $\ell=30$ sub-blocks of which $\tau=6$ sub-blocks are used for channel estimation, $K=6$ RIS elements, $A=2$ available phase shifts, a symbol-toRIS control rate $m=1$, an average power constraint of $P=10 \mathrm{~dB}$, and an input constellation given by 2-ASK $\mathcal{S}=\{\sigma, 3 \sigma\}$ with $\sigma=1 / \sqrt{5}$. While both capacity and rate achieved by the max-SNR scheme increase with the number of receive antennas, the effect is more prominent for joint encoding since, for the max-SNR scheme, spatial multiplexing is restricted by the number of transmit antennas, whereas, for joint encoding, spatial multiplexing is restricted by the number of RIS elements.

Layered Encoding. In Fig. 8, we compare the rate achieved by layered encoding to that of the max-SNR method and to the capacity by plotting the lower bounds on the rate as a function of the average power $P$, with $\ell=50$ sub-blocks of which $\tau=3$ sub-blocks are used for channel estimation, $N=2$ receive antennas, $K=3$ RIS elements, $A=2$ available phase shifts, a symbol-to-RIS control rate $m=2$, and input constellation given by 4 -ASK or QPSK $\mathcal{S}=\{ \pm 1, \pm i\}$. For layered encoding, we set $\mu=1$ pilot, which was seen to 


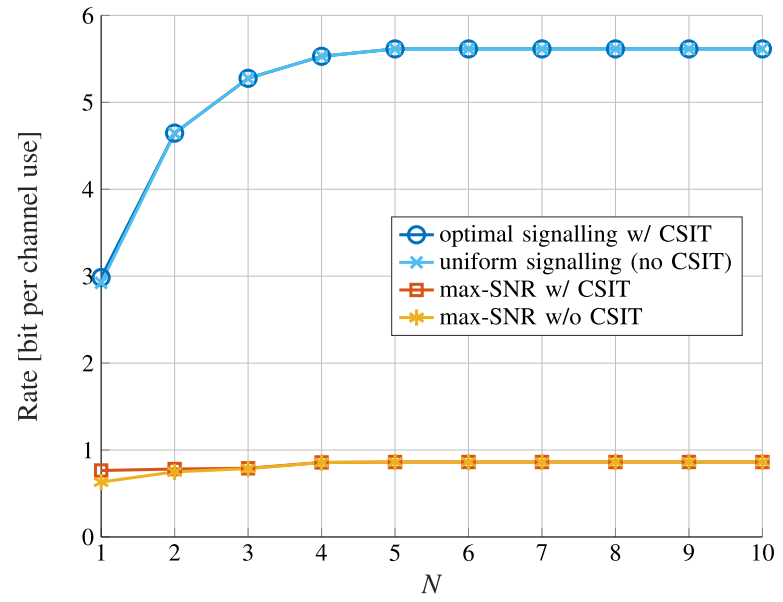

Fig. 7. Rate lower bounds as a function of the number of receive antennas $N$ for $\ell=30, \tau=6, K=6, A=2, m=1, P=10 \mathrm{~dB}$, and 2-ASK input constellation.

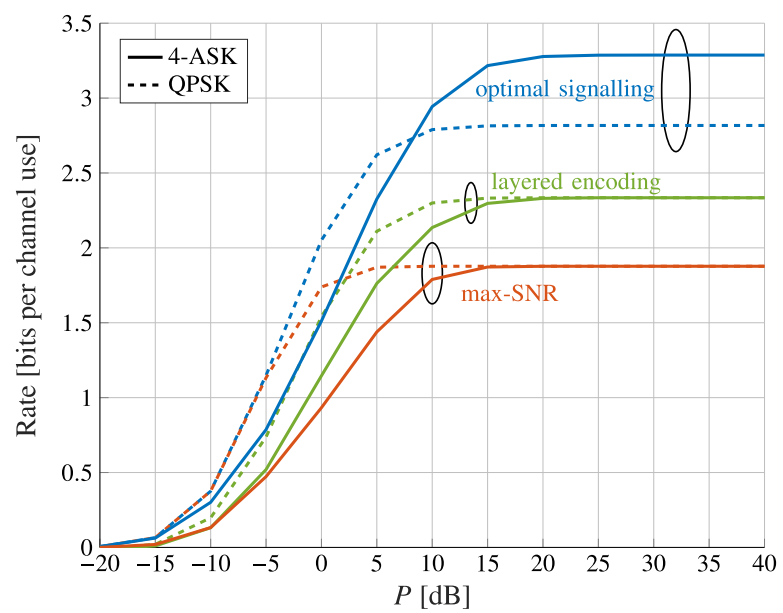

Fig. 8. Rate lower bounds as a function of the normalized power $P[\mathrm{~dB}]$ for $\ell=50, \tau=3, N=2, K=3, A=2, m=2, \mu=1$, and 4-ASK or QPSK input constellation.

maximize the rate in this experiment. It is observed that, for sufficiently high SNR, the layered-encoding scheme improves the rate over the max-SNR approach, whereas, for low SNR and QPSK input constellation, the max-SNR approach is favorable. This is because layered encoding is beneficial when the rate gained from modulating the reflection pattern is higher than the rate loss caused by fixing additional pilot symbols. Note that, in the high-SNR regime, as apparent from the limits in (39) and (54), layered encoding achieves a higher rate when $K \log _{2}(A)>\mu \log _{2}(S)$. In addition, while PSK outperforms ASK when used with the max-SNR and layered-encoding schemes, the opposite is true with joint encoding in the highSNR regime. In fact, as discussed in Proposition 1, in the high-SNR regime, out of all finite input sets $\mathcal{S}$ with the same size, ASK achieves the maximum capacity.

On the RIS control rate. The gain of using the state of the RIS as a medium for conveying information is expected to decrease as the rate of the control link from the transmitter to the RIS decreases. This is illustrated in Fig. 9, where we plot the rate with perfect CSI at both the transmitter and receiver as a function of the RIS control rate factor $m$, with $N=2$

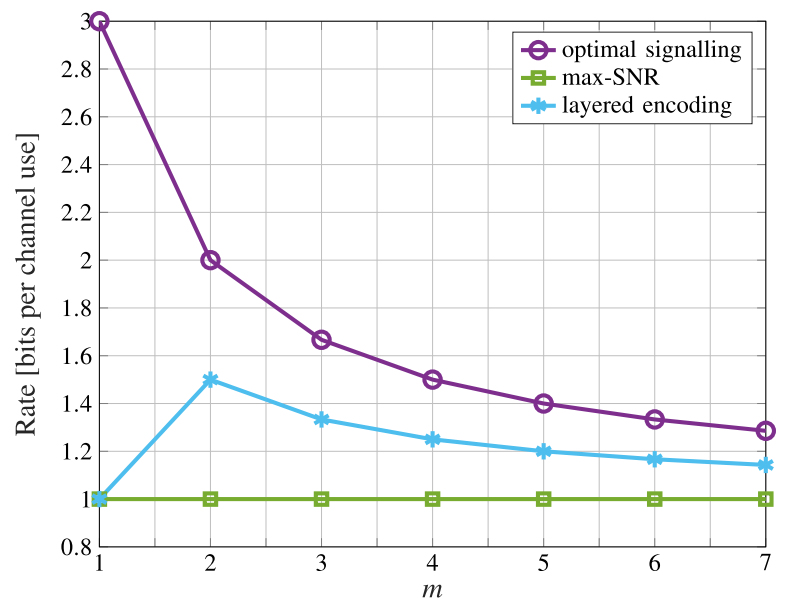

Fig. 9. Rates with perfect CSI as a function of the RIS control rate factor $m$ for $N=2, K=2, A=2, P=40 \mathrm{~dB}, \mu=1$, and 2-ASK input constellation.

receive antennas, $K=2$ RIS elements, $A=2$ available phase shifts, an average power constraint of $P=40 \mathrm{~dB}$, and an input constellation 2-ASK. Note that the performance of the layeredencoding scheme improves from $m=1$ to $m=2$ since, for $m=1$, the transmitted symbol in each sub-block is used as a pilot, and hence only the first layer carries information. It is observed that joint encoding achieves three times the rate of max-SNR for $m=1$, but the gain reduces to a factor of 1.3 for $m=7$.

\section{CONCLUSION}

In this work, we have studied the capacity of an RISaided system. We focused on a fundamental model with one transmitter and one receiver, where the CSI is acquired through pilot-assisted channel estimation. The common approach of using the RIS as a passive beamformer to maximize the achievable rate was shown to be generally suboptimal in terms of the achievable rate for finite input constellations, especially for slowly-changing channels. Instead, the capacity-achieving scheme was proved to jointly encode information in the RIS reflection pattern as well as in the transmitted signal. While the optimal scheme was shown to require a more accurate channel estimation compared to the max-SNR approach, the gain of encoding information in the reflection pattern of the RIS was demonstrated to be significant for a sufficiently high RIS control rate. In addition, a suboptimal, yet practical, strategy based on separate layered encoding and successive cancellation decoding was demonstrated to outperform passive beamforming for sufficiently high SNR levels, and motivates RIS-based modulation design [8]-[14] for single-RF MIMO communication.

Among related problems left open by this study, we mention the design of low-complexity joint encoding and decoding strategies that approach capacity; the derivation of the capacity for channels with direct link from the transmitter to the receiver and a fading link from the transmitter to the RIS, for noisy RIS [35], and for RIS with mutual coupling [24]; as well as extensions to RIS systems with multiple users/surfaces [36] or with security constraints [37]. Another related problem is finding the optimal input distribution for a slowly fading channel with CSI only at the receiver [38]. 


\section{APPENDIX}

\section{A. Proof of Proposition 1}

The model in (22) can be viewed as a standard channel with input $\mathbf{X}$, output $\mathbf{y}$, and known CSI $\hat{\mathbf{h}}$. This is because the transmitter directly controls the states of the RIS $\boldsymbol{\theta}_{i}(t)$ and the transmitted symbols $\mathbf{s}_{i}(t)$ for $i \in[\ell]$ and $t \in[n / m]$. Therefore, it follows, from the channel coding theorem [32, Ch. 7], [39, Ch. 7.4.1], that the ergodic capacity can be expressed as

$$
C\left(\tau, \gamma_{\tau}, \boldsymbol{X}_{1: \tau}\right)=\max _{\substack{p_{\mathbf{X} \mid \hat{\mathbf{h}}}(\boldsymbol{X} \mid \hat{\boldsymbol{h}}): \\ \mathbb{E}\left[\operatorname{tr}\left(\mathbf{X X}^{*}\right)\right] \leq K m(\ell-\tau), \mathbf{X} \in \mathcal{C}^{1 \times(\ell-\tau)}}} \frac{1}{m \ell} I(\mathbf{X} ; \mathbf{y} \mid \hat{\mathbf{h}}) .
$$

The mutual information $I(\mathbf{X} ; \mathbf{y} \mid \hat{\mathbf{h}})$ in (65) can be written as $I(\mathbf{X} ; \mathbf{y} \mid \hat{\mathbf{h}})=h(\mathbf{y} \mid \hat{\mathbf{h}})-h(\mathbf{y} \mid \hat{\mathbf{h}}, \mathbf{X})$. In addition, the conditional probability density function of the output $\mathbf{y}$ given the estimate $\hat{\mathbf{h}}$ and input $\mathbf{X}$ is

$$
\begin{aligned}
& p_{\mathbf{y} \mid \hat{\mathbf{h}}, \mathbf{X}}(\boldsymbol{y} \mid \hat{\boldsymbol{h}}, \boldsymbol{X}) \\
& \quad=\frac{1}{\pi^{N m(\ell-\tau)|\boldsymbol{\Gamma}(\boldsymbol{X})|}} \exp \left(-\left\|\boldsymbol{y}-\gamma_{d} \boldsymbol{X}^{\otimes} \hat{\boldsymbol{h}}\right\|_{\boldsymbol{\Gamma}(\boldsymbol{X})}^{2}\right),
\end{aligned}
$$

where the covariance matrix $\boldsymbol{\Gamma}(\boldsymbol{X})$ is defined in (27). Therefore, the conditional differential entropy $h(\mathbf{y} \mid \hat{\mathbf{h}}, \mathbf{X})$ is given as

$$
\begin{aligned}
& h(\mathbf{y} \mid \hat{\mathbf{h}}, \mathbf{X}) \\
& =N m(\ell-\tau) \log _{2}(\pi e) \\
& \quad+\int_{\mathbb{C}^{N K \times 1}} p_{\hat{\mathbf{h}}}(\hat{\boldsymbol{h}}) \sum_{\mathbf{X} \in \mathcal{C}^{1 \times(\ell-\tau)}} p_{\mathbf{X} \mid \hat{\mathbf{h}}}(\boldsymbol{X} \mid \hat{\boldsymbol{h}}) \log _{2} \operatorname{det}(\boldsymbol{\Gamma}(\boldsymbol{X})) \mathrm{d} \hat{\boldsymbol{h}},
\end{aligned}
$$

and the conditional differential entropy $h(\mathbf{y} \mid \hat{\mathbf{h}})$ can be expressed as (see, e.g., [40, Eq. (3)] and [41, Eq. (4)])

$$
\begin{aligned}
& h(\mathbf{y} \mid \hat{\mathbf{h}}) \\
& =N m(\ell-\tau) \log _{2}(\pi) \\
& \quad-\int_{\mathbb{C}^{N K \times 1}} p_{\hat{\mathbf{h}}}(\hat{\boldsymbol{h}}) \int_{\mathbb{C}^{N m(\ell-\tau) \times 1}} p_{\mathbf{z}}(\boldsymbol{z}) \sum_{\boldsymbol{X}_{1} \in \mathcal{C}^{1 \times(\ell-\tau)}} p_{\mathbf{X} \mid \hat{\mathbf{h}}}\left(\boldsymbol{X}_{1} \mid \hat{\boldsymbol{h}}\right) \\
& \quad \log _{2}\left(\sum_{\boldsymbol{X}_{2} \in \mathcal{C}^{1 \times(\ell-\tau)}} \frac{p_{\mathbf{X} \mid \hat{\mathbf{h}}}\left(\boldsymbol{X}_{2} \mid \hat{\boldsymbol{h}}\right)}{\left|\boldsymbol{\Gamma}\left(\boldsymbol{X}_{1}\right)\right|} e^{u}\right) \mathrm{d} \boldsymbol{z} \mathrm{d} \hat{\boldsymbol{h}}
\end{aligned}
$$

with $\mathbf{z} \sim \mathcal{C N}\left(\mathbf{0}, \boldsymbol{I}_{N m(\ell-\tau)}\right)$ and where we have defined the scalar

$$
\begin{aligned}
u \triangleq & \ln \left(\frac{\left|\boldsymbol{\Gamma}\left(\boldsymbol{X}_{1}\right)\right|}{\left|\boldsymbol{\Gamma}\left(\boldsymbol{X}_{2}\right)\right|}\right) \\
& -\left\|\boldsymbol{V}\left(\boldsymbol{X}_{1}\right) \boldsymbol{z}+\gamma_{d}\left(\boldsymbol{X}_{1}^{\otimes}-\boldsymbol{X}_{2}^{\otimes}\right) \hat{\boldsymbol{h}}\right\|_{\Gamma\left(\boldsymbol{X}_{2}\right)}^{2} .
\end{aligned}
$$

Overall, by subtracting (67) from (68) and applying the conditional CGF definition in (25), we get (29). Note that the mutual information $I(\mathbf{X} ; \mathbf{y} \mid \hat{\mathbf{h}})$ is a concave function of $p_{\mathbf{X} \mid \hat{\mathbf{h}}}(\boldsymbol{X} \mid \hat{\boldsymbol{h}})$ for fixed $p_{\mathbf{y} \mid \hat{\mathbf{h}}, \mathbf{X}}(\boldsymbol{y} \mid \hat{\boldsymbol{h}}, \boldsymbol{X})$ [32, Theorem 2.7.4]. Therefore, problem (65) can be solved using convex optimization tools.

In the high-SNR regime, the channel can be perfectly estimated if $\tau \geq K$, and hence we have the limit

$$
\lim _{P \rightarrow \infty} I(\mathbf{X} ; \mathbf{y} \mid \hat{\mathbf{h}})=H(\mathbf{X} \mid \hat{\mathbf{h}}) \leq H(\mathbf{X}) \leq(\ell-\tau) \log _{2}(|\mathcal{C}|),
$$

where the equality is achieved for a uniform distribution $p_{\mathbf{X} \mid \hat{\mathbf{h}}}(\boldsymbol{X} \mid \hat{\boldsymbol{h}})=1 /|\mathcal{C}|^{\ell-\tau}$. Furthermore, the cardinality of the set $\mathcal{C}$ in (8) is upper bounded as $|\mathcal{C}| \leq S^{m} A^{K}$, where the equality is achieved for the ASK input constellation.

\section{B. Proof of Corollary 2}

For perfect CSI, i.e., $\hat{\mathbf{h}}(t)=\overline{\mathbf{h}}(t)$ and $\tau=0$, the mutual information $I(\mathbf{X} ; \mathbf{y} \mid \hat{\mathbf{h}})$ in (65) can be upper bounded as

$$
\begin{aligned}
& I(\mathbf{X} ; \mathbf{y} \mid \hat{\mathbf{h}})= I(\mathbf{X} ; \mathbf{y} \mid \overline{\mathbf{h}}) \\
&= h\left(\mathbf{y}_{1}, \ldots, \mathbf{y}_{\ell} \mid \overline{\mathbf{h}}\right)-h\left(\mathbf{y}_{1}, \ldots, \mathbf{y}_{\ell} \mid \overline{\mathbf{h}}, \overline{\mathbf{X}}_{1}, \ldots, \overline{\mathbf{X}}_{\ell}\right) \\
& \stackrel{(\text { a) }}{=} \sum_{i=1}^{\ell}\left[h\left(\mathbf{y}_{i} \mid \overline{\mathbf{h}}, \mathbf{y}_{1}, \ldots, \mathbf{y}_{i-1}\right)\right. \\
&\left.-h\left(\mathbf{y}_{i} \mid \overline{\mathbf{h}}, \overline{\mathbf{X}}_{1}, \ldots, \overline{\mathbf{X}}_{\ell}, \mathbf{y}_{1}, \ldots, \mathbf{y}_{i-1}\right)\right] \\
& \stackrel{(\text { b) }}{=} \sum_{i=1}^{\ell}\left[h\left(\mathbf{y}_{i} \mid \overline{\mathbf{h}}, \mathbf{y}_{1}, \ldots, \mathbf{y}_{i-1}\right)-h\left(\mathbf{y}_{i} \mid \overline{\mathbf{h}}, \overline{\mathbf{X}}_{i}\right)\right] \\
& \stackrel{\text { (c) }}{\leq} \sum_{i=1}^{\ell}\left[h\left(\mathbf{y}_{i} \mid \overline{\mathbf{h}}\right)-h\left(\mathbf{y}_{i} \mid \overline{\mathbf{h}}, \overline{\mathbf{X}}_{i}\right)\right] \\
&=\sum_{i=1}^{\ell} I\left(\overline{\mathbf{X}}_{i} ; \mathbf{y}_{i} \mid \overline{\mathbf{h}}\right),
\end{aligned}
$$

where the equality (a) follows from the chain rule for differential entropy [32, Thm. 8.6.2]; the equality (b) holds since, given the channel vector $\overline{\mathbf{h}}$ and input $\overline{\mathbf{X}}_{i}$, the channel output $\mathbf{y}_{i}$ in (11), for sub-block $i \in[\ell]$, is independent of the other channel outputs and inputs; and the inequality (c) is due to the conditioning reduces entropy property [32, Thm. 2.6.5]. Note that the equality (c) is achieved if the sub-blocks are encoded independently, i.e.,

$$
p_{\mathbf{X} \mid \overline{\mathbf{h}}}(\boldsymbol{X} \mid \overline{\boldsymbol{h}})=p_{\mathbf{X} \mid \overline{\mathbf{h}}}\left(\overline{\boldsymbol{X}}_{1}, \ldots, \overline{\boldsymbol{X}}_{\ell} \mid \overline{\boldsymbol{h}}\right)=\prod_{i=1}^{\ell} p_{\overline{\mathbf{X}} \mid \overline{\mathbf{h}}}\left(\overline{\boldsymbol{X}}_{i} \mid \overline{\boldsymbol{h}}\right)
$$

for which we have

$$
I(\mathbf{X} ; \mathbf{y} \mid \overline{\mathbf{h}})=\ell I\left(\overline{\mathbf{X}}_{1} ; \mathbf{y}_{1} \mid \overline{\mathbf{h}}\right) .
$$

Therefore, Corollary 2 is proved by repeating the proof in Appendix A with the caveat that the mutual information $I(\mathbf{X} ; \mathbf{y} \mid \hat{\mathbf{h}})$ in (65) is replaced with $\ell I\left(\overline{\mathbf{X}}_{1} ; \mathbf{y}_{1} \mid \overline{\mathbf{h}}\right)$ and the input is distributed as in (72).

\section{Proof of Proposition 3}

The channel in (45) is equivalent to a point-to-point Gaussian multiple-input multiple-output (MIMO) channel with PSK input Q. Therefore, for layer $w_{1}$, the following rate is achievable

$$
R_{1}\left(\tau, \gamma_{\tau}, \boldsymbol{X}_{1: \tau}, \mu\right)=\frac{1}{m \ell} I(\mathbf{Q} ; \overline{\mathbf{Y}} \mid \hat{\mathbf{H}})=\frac{1}{m \ell} I(\mathbf{Q} ; \overline{\mathbf{y}} \mid \hat{\mathbf{h}}),
$$

where we have defined

$$
\overline{\mathbf{y}} \triangleq \operatorname{vec}(\overline{\mathbf{Y}})=\gamma_{d} \mathbf{Q}^{\otimes} \overline{\mathbf{h}}+\overline{\mathbf{z}}
$$

with $\overline{\mathbf{z}} \sim \mathcal{C N}\left(\mathbf{0}, \boldsymbol{I}_{N(\ell-\tau)}\right)$, and where the phase shifts matrix $\mathbf{Q}(46)$ is uniformly distributed, i.e., $p_{\mathbf{Q}}(\boldsymbol{Q})=1 / A^{K(\ell-\tau)}$ for 
all $Q \in \mathcal{Q}(\ell-\tau)$ (47). It hence follows from the proof of Proposition 1 (Appendix A) that

$$
\begin{aligned}
& I(\mathbf{Q} ; \overline{\mathbf{y}} \mid \hat{\mathbf{h}}) \\
& =-N(\ell-\tau) \log _{2}(e) \\
& \quad-\int_{\mathbb{C}^{N K \times 1}} p_{\hat{\mathbf{h}}}(\hat{\boldsymbol{h}}) \int_{\mathbb{C}^{N(\ell-\tau) \times 1}} p_{\overline{\mathbf{z}}}(\overline{\boldsymbol{z}}) \sum_{\boldsymbol{Q}_{1} \in \mathcal{Q}(\ell-\tau)} \frac{1}{A^{K(\ell-\tau)}} \\
& \quad \log _{2}\left(\sum_{\boldsymbol{Q}_{2} \in \mathcal{Q}(\ell-\tau)} \frac{\exp \left(u_{1}\right)}{A^{K(\ell-\tau)}}\right) \mathrm{d} \overline{\boldsymbol{z}} \mathrm{d} \hat{\boldsymbol{h}},
\end{aligned}
$$

where we have defined the scalar

$$
u_{1} \triangleq \ln \left(\frac{\left|\boldsymbol{\Gamma}\left(\boldsymbol{Q}_{1}\right)\right|}{\left|\boldsymbol{\Gamma}\left(\boldsymbol{Q}_{2}\right)\right|}\right)-\left\|\boldsymbol{V}\left(\boldsymbol{Q}_{1}\right) \overline{\boldsymbol{z}}+\gamma_{d}\left(\boldsymbol{Q}_{1}^{\otimes}-\boldsymbol{Q}_{2}^{\otimes}\right) \hat{\boldsymbol{h}}\right\|_{\boldsymbol{\Gamma}\left(\boldsymbol{Q}_{2}\right)}^{2} .
$$

By applying the conditional CGF definition in (25) to the achievable rate in (74), we get (49) with the aid of (76).

For layer $w_{2}$, let $\check{\mathbf{Y}}_{i}(t)$ denote the last $(m-\mu)$ columns of $\mathbf{Y}_{i}(t)(7)$, i.e.,

$$
\begin{aligned}
\check{\mathbf{Y}}_{i}(t) & \triangleq\left(\mathbf{y}_{i, \mu+1}(t), \ldots, \mathbf{y}_{i, m}(t)\right) \\
& =\gamma_{d} \mathbf{H}(t) e^{j \boldsymbol{\theta}_{i}(t)} \check{\mathbf{s}}_{i}^{\top}(t)+\check{\mathbf{Z}}_{i}(t),
\end{aligned}
$$

where we have defined $\check{\mathbf{s}}_{i}(t) \triangleq\left(\mathrm{s}_{i, \mu+1}, \ldots, \mathrm{s}_{i, m}\right)^{\top} \in$ $\mathcal{S}^{(m-\mu) \times 1}$ and $\check{\mathbf{Z}}_{i}(t) \in \mathbb{C}^{N \times(m-\mu)}$ whose elements are i.i.d. as $\mathcal{C N}(0,1)$. After layer $w_{1}$ is decoded, the receiver reconstructs the phase shifts $\left\{\boldsymbol{\theta}_{i}(t)\right\}_{i}, i=\tau+1, \ldots, \ell, t \in[n / T]$, and decodes layer $w_{2}$ from the received signals

$$
\check{\mathbf{Y}}(t) \triangleq\left(\check{\mathbf{Y}}_{\tau+1}(t), \ldots, \check{\mathbf{Y}}_{\ell}(t)\right)=\gamma_{d} \mathbf{H}(t) \check{\mathbf{X}}(t)+\check{\mathbf{Z}}(t),
$$

where we have defined $\check{\mathbf{Z}}(t) \triangleq\left(\check{\mathbf{Z}}_{\tau+1}(t), \ldots, \check{\mathbf{Z}}_{\ell}(t)\right)$ and

$$
\check{\mathbf{X}}(t) \triangleq\left(e^{j \boldsymbol{\theta}_{\tau+1}(t)} \check{\mathbf{s}}_{\tau+1}^{\top}(t), \ldots, e^{j \boldsymbol{\theta}_{\ell}(t)} \check{\mathbf{s}}_{\ell}^{\boldsymbol{\top}}(t)\right) \in \mathcal{C}(\boldsymbol{\Theta} ; \mu)
$$

with $\boldsymbol{\Theta} \triangleq\left(\boldsymbol{\theta}_{\tau+1}, \ldots, \boldsymbol{\theta}_{\tau+1}\right)$. Therefore, the following rate is achievable for layer $w_{2}$

$$
\begin{aligned}
R_{2}\left(\tau, \gamma_{\tau}, \boldsymbol{X}_{1: \tau}, \mu\right) & =\frac{1}{m \ell} I\left(\check{\mathbf{X}} ; \check{\mathbf{Y}} \mid \hat{\mathbf{h}}, \boldsymbol{\theta}_{\tau+1}, \ldots, \boldsymbol{\theta}_{\ell}\right) \\
& =\frac{1}{m \ell} I\left(\check{\mathbf{X}} ; \check{\mathbf{y}} \mid \hat{\mathbf{h}}, \boldsymbol{\theta}_{\tau+1}, \ldots, \boldsymbol{\theta}_{\ell}\right),
\end{aligned}
$$

where we have defined

$$
\check{\mathbf{y}} \triangleq \operatorname{vec}(\check{\mathbf{Y}})=\gamma_{d} \check{\mathbf{X}}^{\otimes} \overline{\mathbf{h}}+\check{\mathbf{z}}
$$

with $\check{\mathbf{z}} \sim \mathcal{C N}\left(\mathbf{0}, \boldsymbol{I}_{N(m-\mu)(\ell-\tau)}\right)$, and where the input $\check{\mathbf{X}}$ is uniformly distributed, i.e., $p_{\check{\mathbf{X}}}(\check{\boldsymbol{X}})=1 / S^{(m-\mu)(\ell-\tau)}$ for all $\check{\boldsymbol{X}} \in \mathcal{C}(\boldsymbol{\Theta} ; \mu)$ (53). Similar to layer $w_{1}$, we have

$$
\begin{aligned}
& I\left(\check{\mathbf{X}} ; \check{\mathbf{y}} \mid \hat{\mathbf{h}}, \boldsymbol{\theta}_{\tau+1}, \ldots, \boldsymbol{\theta}_{\ell}\right) \\
& =-N(m-\mu)(\ell-\tau) \log _{2}(e) \\
& \quad-\int_{\mathbb{C}^{N K \times 1}} p_{\hat{\mathbf{h}}}(\hat{\boldsymbol{h}}) \int_{\mathbb{C}^{N(\ell-\tau) \times 1}} p_{\check{\check{z}}(\check{\boldsymbol{z}})} \sum_{\boldsymbol{\Theta} \in \mathcal{A}^{K \times(\ell-\tau)}} \frac{1}{A^{K(\ell-\tau)}} \\
& \quad \sum_{\check{\boldsymbol{X}}_{1} \in C(\boldsymbol{\Theta})} \frac{1}{S^{(m-\mu)(\ell-\tau)}} \log _{2}\left(\sum_{\check{\boldsymbol{X}}_{2} \in C(\boldsymbol{\Theta})} \frac{\exp \left(u_{2}\right)}{S^{(m-\mu)(\ell-\tau)}}\right) \mathrm{d} \check{\boldsymbol{z}} \mathrm{d} \hat{\boldsymbol{h}},
\end{aligned}
$$

where we have defined the scalar

$$
\begin{aligned}
u_{2} \triangleq & \ln \left(\frac{\left|\boldsymbol{\Gamma}\left(\check{\boldsymbol{X}}_{1}\right)\right|}{\left|\boldsymbol{\Gamma}\left(\check{\boldsymbol{X}}_{2}\right)\right|}\right) \\
& -\left\|\boldsymbol{V}\left(\check{\boldsymbol{X}}_{1}\right) \check{\boldsymbol{z}}+\gamma_{d}\left(\check{\boldsymbol{X}}_{1}^{\otimes}-\check{\boldsymbol{X}}_{2}^{\otimes}\right) \hat{\boldsymbol{h}}\right\|_{\boldsymbol{\Gamma}\left(\check{\boldsymbol{X}}_{2}\right)}^{2} .
\end{aligned}
$$

By applying the conditional CGF definition in (25) to the achievable rate in (81), we get (51) with the aid of (83).

As in the proof of Proposition 1, for $\tau \geq K$, we have the high-SNR limits

$$
\lim _{P \rightarrow \infty} I(\mathbf{Q} ; \overline{\mathbf{y}} \mid \hat{\mathbf{h}})=H(\mathbf{Q})=(\ell-\tau) K \log _{2}(A)
$$

and

$$
\begin{aligned}
\lim _{P \rightarrow \infty} I\left(\check{\mathbf{X}} ; \check{\mathbf{y}} \mid \hat{\mathbf{h}}, \boldsymbol{\theta}_{\tau+1}, \ldots, \boldsymbol{\theta}_{\ell}\right) & =H\left(\check{\mathbf{X}} \mid \boldsymbol{\theta}_{\tau+1}, \ldots, \boldsymbol{\theta}_{\ell}\right) \\
& =(\ell-\tau)(m-\mu) \log _{2}(S) .
\end{aligned}
$$

\section{Proof of Proposition 4}

To lower bound the capacity, we restrict the input distribution $p_{\mathbf{X} \mid \hat{\mathbf{h}}}(\boldsymbol{X} \mid \hat{\boldsymbol{h}})$ in (65) such that each sub-block is encoded independently, given the channel estimate, as in (72). Furthermore, we lower bound the mutual information $I(\mathbf{X} ; \mathbf{y} \mid \hat{\mathbf{h}})$ in (65) as

$$
\begin{aligned}
& I(\mathbf{X} ; \mathbf{y} \mid \hat{\mathbf{h}}) \\
& =H\left(\overline{\mathbf{X}}_{\tau+1}, \ldots, \overline{\mathbf{X}}_{\ell} \mid \hat{\mathbf{h}}\right) \\
& -H\left(\overline{\mathbf{X}}_{\tau+1}, \ldots, \overline{\mathbf{X}}_{\ell} \mid \hat{\mathbf{h}}, \mathbf{y}_{\tau+1}, \ldots, \mathbf{y}_{\ell}\right) \\
& \stackrel{(\text { a) }}{=} \sum_{i=\tau+1}^{\ell}\left[H\left(\overline{\mathbf{X}}_{i} \mid \hat{\mathbf{h}}\right)\right. \\
& \left.-H\left(\overline{\mathbf{X}}_{i} \mid \hat{\mathbf{h}}, \mathbf{y}_{\tau+1}, \ldots, \mathbf{y}_{\ell}, \overline{\mathbf{X}}_{\tau+1}, \ldots, \overline{\mathbf{X}}_{i-1}\right)\right] \\
& \stackrel{\text { (b) }}{\geq} \sum_{i=\tau+1}^{\ell}\left[H\left(\overline{\mathbf{X}}_{i} \mid \hat{\mathbf{h}}\right)-H\left(\overline{\mathbf{X}}_{i} \mid \hat{\mathbf{h}}, \mathbf{y}_{i}\right)\right] \\
& =(\ell-\tau) I\left(\overline{\mathbf{X}}_{\tau+1} ; \mathbf{y}_{\tau+1} \mid \hat{\mathbf{h}}\right),
\end{aligned}
$$

where the equality (a) follows from the entropy chain rule [32, Thm. 2.5.1] and the input distribution in (72); and the inequality (b) is due to the conditioning reduces entropy property [32, Thm. 2.6.5]. Proposition 4 is then proved by repeating the proof in Appendix A with the caveat that the mutual information $I(\mathbf{X} ; \mathbf{y} \mid \hat{\mathbf{h}})$ is replaced with the lower bound (87). Note that, for $\hat{\mathbf{h}}=\overline{\mathbf{h}}$ and $\tau=0$, the lower bound in (87) reduces to (73). That is, for perfect CSI, the lower bound coincides with the capacity.

\section{E. Proof of Proposition 5}

Similar to the proof of Proposition 4 (Appendix D), the mutual information $I(\mathbf{Q} ; \overline{\mathbf{y}} \mid \mathbf{h})$ in (74) can be lower bounded as

$$
\begin{aligned}
I(\mathbf{Q} ; \overline{\mathbf{y}} \mid \hat{\mathbf{h}}) \stackrel{(\mathrm{a})}{=} I\left(\boldsymbol{\theta}_{\tau+1}, \ldots, \boldsymbol{\theta}_{\ell} ; \overline{\mathbf{y}}_{\tau+1}, \ldots, \overline{\mathbf{y}}_{\ell} \mid \hat{\mathbf{h}}\right) \\
\\
\geq(\ell-\tau) I\left(\boldsymbol{\theta}_{\tau+1} ; \overline{\mathbf{y}}_{\tau+1} \mid \hat{\mathbf{h}}\right),
\end{aligned}
$$


where the equality (a) follows from the definitions in (44), (46), and (75). Furthermore, the mutual information $I\left(\check{\mathbf{X}} ; \check{\mathbf{y}} \mid \hat{\mathbf{h}}, \boldsymbol{\theta}_{\tau+1}, \ldots, \boldsymbol{\theta}_{\ell}\right)$ in (81) can be lower bounded as

$$
\begin{aligned}
& I\left(\check{\mathbf{X}} ; \check{\mathbf{y}} \mid \hat{\mathbf{h}}, \boldsymbol{\theta}_{\tau+1}, \ldots, \boldsymbol{\theta}_{\ell}\right) \\
& \quad \stackrel{(\mathrm{a})}{=} I\left(\check{\mathbf{s}}_{\tau+1}, \ldots, \check{\mathbf{s}}_{\ell} ; \check{\mathbf{Y}}_{\tau+1}, \ldots, \check{\mathbf{Y}}_{\ell} \mid \hat{\mathbf{h}}, \boldsymbol{\theta}_{\tau+1}, \ldots, \boldsymbol{\theta}_{\ell}\right) \\
& \quad \geq(\ell-\tau) I\left(\check{\mathbf{s}}_{\tau+1} ; \check{\mathbf{Y}}_{\tau+1} \mid \hat{\mathbf{h}}, \boldsymbol{\theta}_{\tau+1}\right),
\end{aligned}
$$

where the equality (a) follows from the definitions in (79), (80), and (82). Proposition 5 is then proved by repeating the proof in Appendix $\mathrm{C}$ with the caveat that the mutual information $I(\mathbf{Q} ; \overline{\mathbf{y}} \mid \hat{\mathbf{h}})$ is replaced with the lower bound in (88), and the mutual information $I\left(\check{\mathbf{X}} ; \check{\mathbf{y}} \mid \hat{\mathbf{h}}, \boldsymbol{\theta}_{\tau+1}, \ldots, \boldsymbol{\theta}_{\ell}\right)$ is replaced with the lower bound in (89).

\section{REFERENCES}

[1] M. Di Renzo et al., "Smart radio environments empowered by reconfigurable intelligent surfaces: How it works, state of research, and the road ahead," IEEE J. Sel. Areas Commun., vol. 38, no. 11, pp. 2450-2525, Nov. 2020.

[2] M. Di Renzo et al., "Reconfigurable intelligent surfaces vs. relaying: Differences, similarities, and performance comparison," IEEE Open J. Commun. Soc., vol. 1, pp. 798-807, 2020.

[3] Q. Wu and R. Zhang, "Towards smart and reconfigurable environment: Intelligent reflecting surface aided wireless network," IEEE Commun. Mag., vol. 58, no. 1, pp. 106-112, Jan. 2019.

[4] Y. Liu et al., "Reconfigurable intelligent surfaces: Principles and opportunities," 2020, arXiv:2007.03435. [Online]. Available: http://arxiv.org/abs/2007.03435

[5] M. D. Renzo et al., "Smart radio environments empowered by reconfigurable AI meta-surfaces: An idea whose time has come," EURASIP J. Wireless Commun. Netw., vol. 2019, no. 1, pp. 1-20, Dec. 2019.

[6] X. Yuan et al., "Reconfigurable-intelligent-surface empowered wireless communications: Challenges and opportunities," IEEE Wireless Commun., vol. 28, no. 2, pp. 136-143, Apr. 2021.

[7] Q. Wu et al., "Intelligent reflecting surface aided wireless communications: A tutorial," IEEE Trans. Commun., vol. 69, no. 5, pp. 3313-3351, May 2021.

[8] Q. Li, M. Wen, and M. Di Renzo, "Single-RF MIMO: From spatial modulation to metasurface-based modulation," 2020, arXiv:2009.00789. [Online]. Available: http://arxiv.org/abs/2009.00789

[9] W. Tang et al., "Wireless communications with programmable metasurface: New paradigms, opportunities, and challenges on transceiver design," IEEE Wireless Commun., vol. 27, no. 2, pp. 180-187, Apr. 2020.

[10] E. Basar, "Media-based modulation for future wireless systems: A tutorial," IEEE Wireless Commun., vol. 26, no. 5, pp. 160-166, Oct. 2019.

[11] W. Yan, X. Yuan, Z.-Q. He, and X. Kuai, "Passive beamforming and information transfer design for reconfigurable intelligent surfaces aided multiuser MIMO systems," IEEE J. Sel. Areas Commun., vol. 38, no. 8, pp. 1793-1808, Aug. 2020.

[12] W. Yan, X. Yuan, and X. Kuai, "Passive beamforming and information transfer via large intelligent surface," IEEE Wireless Commun. Lett., vol. 9, no. 4, pp. 533-537, Apr. 2020.

[13] S. Lin, B. Zheng, G. C. Alexandropoulos, M. Wen, M. Di Renzo, and F. Chen, "Reconfigurable intelligent surfaces with reflection pattern modulation: Beamforming design and performance analysis," IEEE Trans. Wireless Commun., vol. 20, no. 2, pp. 741-754, Feb. 2021.

[14] E. Basar, "Reconfigurable intelligent surface-based index modulation: A new beyond MIMO paradigm for 6G," IEEE Trans. Commun., vol. 68 , no. 5, pp. 3187-3196, May 2020.

[15] T. L. Jensen and E. De Carvalho, "An optimal channel estimation scheme for intelligent reflecting surfaces based on a minimum variance unbiased estimator," in Proc. IEEE Int. Conf. Acoust., Speech Signal Process. (ICASSP), May 2020, pp. 5000-5004.

[16] Q. Wu and R. Zhang, "Intelligent reflecting surface enhanced wireless network: Joint active and passive beamforming design," in Proc. IEEE Global Commun. Conf. (GLOBECOM), Dec. 2018, pp. 1-6.

[17] N. S. Perović, L. N. Tran, M. Di Renzo, and M. F. Flanagan, "Achievable rate optimization for MIMO systems with reconfigurable intelligent surfaces," IEEE Trans. Wireless Commun., vol. 20, no. 6, pp. 3865-3882, Jun. 2021.
[18] S. Zhang and R. Zhang, "Capacity characterization for intelligent reflecting surface aided MIMO communication," IEEE J. Sel. Areas Commun., vol. 38, no. 8, pp. 1823-1838, Aug. 2020.

[19] C. You, B. Zheng, and R. Zhang, "Channel estimation and passive beamforming for intelligent reflecting surface: Discrete phase shift and progressive refinement," IEEE J. Sel. Areas Commun., vol. 38, no. 11, pp. 2604-2620, Nov. 2020.

[20] A. Zappone, M. Di Renzo, F. Shams, X. Qian, and M. Debbah, "Overhead-aware design of reconfigurable intelligent surfaces in smart radio environments," IEEE Trans. Wireless Commun., vol. 20, no. 1, pp. 126-141, Jan. 2021.

[21] M.-M. Zhao, Q. Wu, M.-J. Zhao, and R. Zhang, "Intelligent reflecting surface enhanced wireless network: Two-timescale beamforming optimization," IEEE Trans. Wireless Commun., vol. 20, no. 1, pp. 2-17, Jan. 2021.

[22] A. K. Khandani, "Media-based modulation: A new approach to wireless transmission," in Proc. IEEE Int. Symp. Inform. Theory (ISIT), Jul. 2013 pp. 3050-3054

[23] R. Karasik, O. Simeone, M. Di Renzo, and S. Shamai (Shitz), "Beyond max-SNR: Joint encoding for reconfigurable intelligent surfaces," in Proc. IEEE Int. Symp. Inf. Theory (ISIT), Jun. 2020, pp. 2965-2970.

[24] G. Gradoni and M. Di Renzo, "End-to-end mutual coupling aware communication model for reconfigurable intelligent surfaces: An electromagnetic-compliant approach based on mutual impedances," IEEE Wireless Commun. Lett., vol. 10, no. 5, pp. 938-942, May 2021. [Online]. Available: https://ieeexplore.ieee.org/document/9319694, doi: 10.1109/LWC.2021.3050826.

[25] B. Hassibi and B. M. Hochwald, "How much training is needed in multiple-antenna wireless links?" IEEE Trans. Inf. Theory, vol. 49, no. 4, pp. 951-963, Apr. 2003.

[26] S. M. Kay, Fundamentals of Statistical Signal Processing. Upper Saddle River, NJ, USA: Prentice-Hall, 1993.

[27] M.-M. Zhao, Q. Wu, M.-J. Zhao, and R. Zhang, "Exploiting amplitude control in intelligent reflecting surface aided wireless communication with imperfect CSI," IEEE Trans. Commun., vol. 69, no. 6, pp. 4216-4231, Jun. 2021

[28] M. Médard, "The effect upon channel capacity in wireless communications of perfect and imperfect knowledge of the channel," IEEE Trans. Inf. Theory, vol. 46, no. 3, pp. 933-946, May 2000.

[29] R. Bustin, H. V. Poor, and S. Shamai (Shitz), "Worst additive noise: An information-estimation view," in Proc. IEEE 28th Conv. Elect. Electron. Eng. Israel (IEEEI), Dec. 2014, pp. 1-4.

[30] S. Shamai and S. Verdu, "Worst-case power-constrained noise for binaryinput channels," IEEE Trans. Inf. Theory, vol. 38, no. 5, pp. 1494-1511, Sep. 1992.

[31] G. Kramer, M. Gastpar, and P. Gupta, "Cooperative strategies and capacity theorems for relay networks," IEEE Trans. Inf. Theory, vol. 51, no. 9, pp. 3037-3063, Sep. 2005.

[32] T. M. Cover and J. A. Thomas, Elements of Information Theory, 2nd ed. Hoboken, NJ, USA: Wiley, 2006.

[33] S. Mohamed, M. Rosca, M. Figurnov, and A. Mnih, "Monte carlo gradient estimation in machine learning," J. Mach. Learn. Res., vol. 21, no. 132 , pp. 1-62, 2020.

[34] M. C. Valenti and X. Xiang, "Constellation shaping for bit-interleaved LDPC coded APSK," IEEE Trans. Commun., vol. 60, no. 10, pp. 2960-2970, Oct. 2012.

[35] X. Qian et al., "Beamforming through reconfigurable intelligent surfaces in single-user MIMO systems: SNR distribution and scaling laws in the presence of channel fading and phase noise," IEEE Wireless Commun. Lett., vol. 10, no. 1, pp. 77-81, Sep. 2021.

[36] H. Guo, Y. C. Liang, J. Chen, and E. G. Larsson, "Weighted sumrate maximization for intelligent reflecting surface enhanced wireless networks," in Proc. IEEE Global Conf. Commun. (GLOBECOM), Dec. 2019, pp. 1-6.

[37] X. Guan, Q. Wu, and R. Zhang, "Intelligent reflecting surface assisted secrecy communication: Is artificial noise helpful or not?" IEEE Wireless Commun. Lett., vol. 9, no. 6, pp. 778-782, Jun. 2020.

[38] S. Shamai and A. Steiner, "A broadcast approach for a single-user slowly fading MIMO channel," IEEE Trans. Inf. Theory, vol. 49, no. 10, pp. 2617-2635, Oct. 2003.

[39] A. El Gamal and Y.-H. Kim, Network Information Theory. Cambridge, U.K.: Cambridge Univ. Press, 2011.

[40] G. Ungerboeck, "Channel coding with multilevel/phase signals," IEEE Trans. Inf. Theory, vol. IT-28, no. 1, pp. 55-67, Jan. 1982.

[41] W. He and C. N. Georghiades, "Computing the capacity of a MIMO fading channel under PSK signaling," IEEE Trans. Inf. Theory, vol. 51, no. 5, pp. 1794-1803, May 2005. 


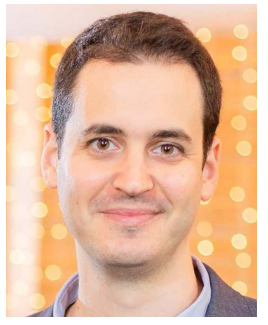

Roy Karasik (Graduate Student Member, IEEE) received the B.Sc. (summa cum laude) and M.Sc. (Hons.) degrees in electrical engineering from the Technion-Israel Institute of Technology, Haifa, Israel, in 2011 and 2014, respectively, where he is currently pursuing the Ph.D. degree. From 2013 to 2017, he worked as an Engineer with Israeli Defense Forces. His research interests include information theory and wireless communications.

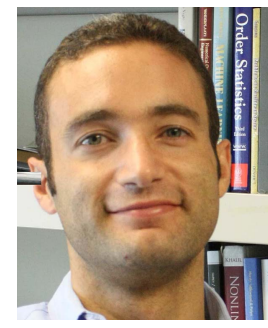

Osvaldo Simeone (Fellow, IEEE) received the M.Sc. (Hons.) and Ph.D. degrees in information engineering from the Politecnico di Milano, Milan, Italy, in 2001 and 2005, respectively. From 2006 to 2017, he was a Faculty Member of the Electrical and Computer Engineering (ECE) Department, New Jersey Institute of Technology (NJIT), where he was affiliated with the Center for Wireless Information Processing (CWiP). He is currently a Professor of information engineering with the Centre for Telecommunications Research, Department of Engineering, King's College London, where he directs the King's Communications, Learning and Information Processing Laboratory. His research has been supported by the U.S. NSF, the ERC, the Vienna Science and Technology Fund, as well as by a number of industrial collaborations. He is the coauthor of two monographs, two edited books published by Cambridge University Press, and more than 150 research journal articles. His research interests include information theory, machine learning, wireless communications, and neuromorphic computing. He was a co-recipient of the IEEE Vehicular Technology Society 2021 Jack Neubauer Memorial Award, the 2019 IEEE Communication Society Best Tutorial Paper Award, the 2018 IEEE Signal Processing Best Paper Award, the 2017 JCN Best Paper Award, the 2015 IEEE Communication Society Best Tutorial Paper Award, and of the Best Paper Awards of IEEE SPAWC 2007 and IEEE WRECOM 2007. He was awarded a Consolidator Grant by the European Research Council (ERC) in 2016. He also serves in the Editorial Board of IEEE Signal Processing Magazine. He is the Chair of the Signal Processing for Communications and Networking Technical Committee of the IEEE Signal Processing Society. He was a Distinguished Lecturer of the IEEE Information Theory Society in 2017 and 2018. He is also a Distinguished Lecturer of the IEEE Communications Society.

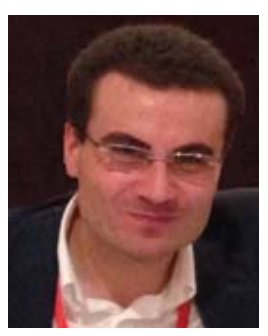

Marco Di Renzo (Fellow, IEEE) received the Laurea (cum laude) and Ph.D. degrees in electrical engineering from the University of L'Aquila, Italy, in 2003 and 2007, respectively, and the Habilitation à Diriger des Recherches (Doctor of Science) degree from University Paris-Sud (now Paris-Saclay University), France, in 2013. Since 2010, he has been with the French National Center for Scientific Research (CNRS), where he is currently a CNRS Research Director (CNRS Professor) with the Laboratory of Signals and Systems (L2S) of Paris-Saclay University-CNRS and CentraleSupelec, Paris, France. In ParisSaclay University, he serves as the Coordinator of the Communications and Networks Research Area of the Laboratory of Excellence DigiCosme, and as a member of the Admission and Evaluation Committee of the Ph.D. School on Information and Communication Technologies. He is a fellow of the U.K. Institution of Engineering and Technology (IET), an Ordinary Member of the European Academy of Sciences and Arts (EASA), and an Ordinary Member of the Academia Europaea (AE). He has received several research distinctions, which include the SEE-IEEE Alain Glavieux Award, the IEEE Jack Neubauer Memorial Best Systems Paper Award, the Royal Academy of Engineering Distinguished Visiting Fellowship, the Nokia Foundation Visiting Professorship, the Fulbright Fellowship, and the 2021 EURASIP Journal on Wireless Communications and Networking Best Paper Award. He is the Editorin-Chief of IEEE COMMUNICATIONS LETTERS and a Distinguished Speaker of the IEEE Vehicular Technology Society. From 2017 to 2020, he was a Distinguished Lecturer of the IEEE Vehicular Technology Society and the IEEE Communications Society. He is also a Highly Cited Researcher in 2019.

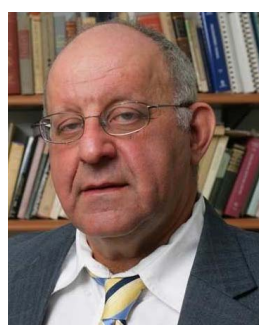

Shlomo Shamai (Shitz) (Life Fellow, IEEE) is currently with the Department of Electrical Engineering, Technion-Israel Institute of Technology, where he is also a Technion Distinguished Professor, and holds the William Fondiller Chair of Telecommunications. He is an URSI Fellow, a member of the Israeli Academy of Sciences and Humanities, and a foreign member of the U.S. National Academy of Engineering. He was a recipient of the 2011 Claude E. Shannon Award, the 2014 Rothschild Prize in Mathematics/Computer Sciences and Engineering, and the 2017 IEEE Richard W. Hamming Medal. He was a co-recipient of the 2018 Third Bell Labs Prize for Shaping the Future of Information and Communications Technology. He was a recipient of numerous technical and paper awards and recognitions of the IEEE (Donald G. Fink Prize Paper Award), Information Theory, Communications and Signal Processing Societies as well as EURASIP. He is listed as a Highly Cited Researcher (Computer Science) for the years 2013, 2014, 2015, 2016, 2017, and 2018. $\mathrm{He}$ has served as an Associate Editor for the Shannon Theory of IEEE TRANSACTIONS ON INFORMATION THEORY, and has also served twice on the Board of Governors of the Information Theory Society. He has also served on the Executive Editorial Board of IEEE TRANSACTIONS ON INFORMATION THEORY, the IEEE Information Theory Society Nominations and Appointments Committee, and the IEEE Information Theory Society, Shannon Award Committee. 